\title{
Durability and Reliability Estimation of Flax Fiber Reinforced Composites Using Tweedie Exponential Dispersion Degradation Process
}

\author{
Weian Yan $\left(\mathbb{D},{ }^{1,2}\right.$ Hassen Riahi $\mathbb{D}^{2},{ }^{2}$ Karim Benzarti $\left(\mathbb{D},{ }^{3}\right.$ Robert Chlela, ${ }^{3}$ Laurence Curtil $\mathbb{D}^{4},{ }^{4}$ \\ and David Bigaud $\mathbb{( D}^{2}$ \\ ${ }^{1}$ College of Transportation and Logistics, East China Jiaotong University, Nanchang, Jiangxi 330013, China \\ ${ }^{2}$ Laboratoire Angevin de Recherche en Ingénierie des Systèmes, LARIS, UNIV Angers, LARIS, SFR MATHSTIC, F-49000 Angers, \\ France \\ ${ }^{3}$ Lab, Univ Gustave Eiffel, ENPC, CNRS, F-77447 Marne-la-Vallée, France \\ ${ }^{4}$ Université Lyon, Université Lyon 1, Laboratoire Matériaux Composites Pour la Construction, LMC2, 82 Bd Niels Bohr, \\ F-69622 Villeurbanne, France
}

Correspondence should be addressed to David Bigaud; david.bigaud@univ-angers.fr

Received 18 November 2020; Revised 16 January 2021; Accepted 23 January 2021; Published 10 February 2021

Academic Editor: Sylwester Samborski

Copyright $(92021$ Weian Yan et al. This is an open access article distributed under the Creative Commons Attribution License, which permits unrestricted use, distribution, and reproduction in any medium, provided the original work is properly cited.

\begin{abstract}
Flax fiber reinforced composites are demonstrating promising outcomes which make them potential candidates to replace synthetic composites in various industrial applications. However, there is limited information regarding their long-term performance, and it is usually acknowledged that natural fibers are less resistant than their synthetic counterparts. In this context, it is crucial to study their durability before considering their use for structural rehabilitation and strengthening in construction. This research aims to study and predict the performance of flax fiber reinforced polymer (FFRP) composites with a biobased epoxy matrix. The test program consists in exposing FFRP laminates and FFRP strengthened concrete slabs to different accelerated ageing conditions over a total period of two years. In the present study, not a single stress variable but various combinations and coupling of two environmental stress variables, temperature $(T)$ and relative humidity $(\mathrm{RH})$, are considered, thereby distinguishing this study from most of the works reported in the literature. Then, a series of mechanical destructive tests are performed periodically on aged samples to evaluate property evolutions over ageing time. The collected experimental data are analyzed to develop a performance evolution model and to evaluate the service lifetime performance of this new biobased FFRP composite. For that, the potential of the Tweedie exponential dispersion (TED) process model, which takes some famous stochastic processes (Wiener process, Gamma process, and inverse Gaussian process) as special cases, is investigated. The TED process modeling, particularly interesting in the cases of complicated degradation mechanisms, is written here for destructive tests and, finally, a reliability analysis based on the TED process model determined is carried out in order to update the FRP design equations provided by international codes in the specific case of FFRP.
\end{abstract}

\section{Introduction}

At this time, flax fiber reinforced polymer (FFRP) composites are still in a research and development stage but are considered a highly promising solution for the future. With their low-carbon footprint and high mechanical properties to weight ratio, FFRPs are poised to be a viable replacement for traditional synthetic composites in specific industrial applications. This transition is due to the shift in international interest towards recyclable and biosourced materials and lowering carbon emissions $[1,2]$. However, a major challenge in using natural fibers remains their sensitivity to environmental conditions, and more specifically in the presence of water $[3,4]$. Numerous studies have investigated the effect of humidity on the mechanical behavior of FFRP laminates, but until now, there are still unanswered 
questions related to the lifetime performance prediction of FFRP and the coupling effects of temperature and humidity on the ageing behavior of these materials.

In civil engineering applications and especially in the domain of structural rehabilitation and strengthening in construction, it is crucial for engineers to get knowledge about the long-term performance of the materials used and not just their short-term characteristics. This extended performance is highly affected by thermal [5] and hygrometric service conditions encountered in the outdoor environment [6-9]. For this reason, this study focusses on a better understanding of temperature and humidity influences on the mechanical properties of FFRP laminates. However, the degradation process of FRP under environmental service conditions is very slow and, thus, is a serious issue if one aims at qualifying the risk of failure of this product over time. Acceleration strategies, by increasing the stress level applied to the product, are used to obtain comprehensive data in a shorter time. While most works on accelerated degradation tests reported in the literature deal with the case of a single stress variable, various combinations of two environmental stress variables, temperature $(T)$ and relative humidity $(\mathrm{RH})$ in the ranges $20^{\circ} \mathrm{C}-60^{\circ} \mathrm{C}$ and $50 \%-100 \%$, respectively, are considered in this article. This enables to quantify the individual and coupling effects of these two stress variables on the parameters of a model enable to describe the loss of FFRP performances over time and to estimate their lifetime.

The premise of performance-based approach is to choose an appropriate degradation model for the composite. There are two large classes of degradation models for degradation analysis, which are the general path models and the stochastic process models. Ye and Xie [10] and Yan et al. [11] provide a general discussion about these approaches. The basic principle of general path modeling is to find a physical parametric model which fits all degradation paths well and to consider some of its parameters to be random to account for variability between samples. For the second class, data are assumed to be generated from a stochastic process. Thus, these latter models can effectively characterize the uncertainty and dynamics of the degradation process. Some studies in the literature, such as [12-17], advocate to apply these stochastic process approaches to model the degradation since statistical indicators related to failure-time are directly obtained by using the properties of the assumed underlying process. It is mainly this feature that motivates the choice of the stochastic process modeling in our study.

There are three stochastic process models mainly represented in the literature: Wiener process, Gamma process, and inverse Gaussian (IG) process. However, these processes are very specific and are not suitable, or generic enough, for products with complicated failure mechanisms and structure [18]. To compensate for that, the potential of the Tweedie exponential dispersion (TED) model, a variant of exponential dispersion models, whose variance function has power exponential form, has been investigated. Actually, the three first processes mentioned are shown to be special cases of TED process with specific values of the exponent in the power form of the variance function $[19,20]$. This ability to model several types of process, to be more generic, is of particular interest for our study since complicated degradation mechanisms are observed. The TED process modeling will be rewritten here for the case of destructive tests whereas its general form found in the literature was first thought for nondestructive tests or continuous degradation monitoring.

The study of the present paper was part of a research project called "MICRO, Matériaux Innovants Composites pour la Réparation d'Ouvrage" (or innovative composite materials for the repairing of civil structures) and funded by ANR (Agence Nationale de la Recherche, or National Research Agency). This project involved a comprehensive durability test program on FFRP strengthening systems which consisted in performing various types of mechanical tests, to follow the evolutions of several performance indicators of these systems (tensile properties and interlaminar shear strength of the FFRP laminates and bond strength of the FFRP/concrete interface) versus exposure time in various accelerated ageing environments. The present paper is only focused on the evolution of the tensile capacity of the FRRP composite over ageing time.

In order to present our main results, the rest of this article is organized as follows: Section 2 will present how the FFRP material is prepared and what are the ageing conditions and the testing procedure. Section 3 will focus on the analysis of accelerated degradation processes. Theoretical aspects about stochastic TED process modeling and lifetime distribution approximation are described first, before explaining how to consider the individual and coupling effects of the two stress variables, temperature and relative humidity, through the generalized Eyring model (GEM), on the parameters of the TED process model. Finally, we will show how to estimate the complete degradation process parameters by maximizing the likelihood function written in the case of destructive tests. It is in Section 4 that we will describe how to carry out the probabilistic prediction of FFRP lifetime based on the TED process modeling of our experimental results. The lifetime will be estimated under the ageing condition $T=20^{\circ} \mathrm{C}-\mathrm{RH}=50 \%$, considered as the reference or service condition. The reliability functions of FFRP will be approximated, in a first attempt, using the safety or environmental coefficients defined for carbon or glass FRP in four different international design codes (since carbon and glass fibers are the only ones considered in the design codes). It will be concluded that current guides are not sufficient and that new design coefficients are necessary for FFRP. These new coefficients are provided at the end of Section 4 in order to address the shortfalls of the current international recommendations. Section 5 will conclude the paper and will give some perspectives of our work.

\section{Materials and Experimental Program}

2.1. Materials and Ageing Conditions. Unidirectional (UD) flax fiber/bioepoxy composites, consisting of 2 layers of $200 \mathrm{~g} / \mathrm{m}^{2}$ UD flax fiber fabrics, were manually prepared using the hand layup technique. The UD FFRP composite plates had a dimension of width $200 \times$ height $350 \mathrm{~mm}$ (see Figure 1(a), later in the article), with a thickness to width ratio lower than 0.01 . The flax fiber fabrics (LINCORE ${ }^{\circledR} \mathrm{FF}$ 


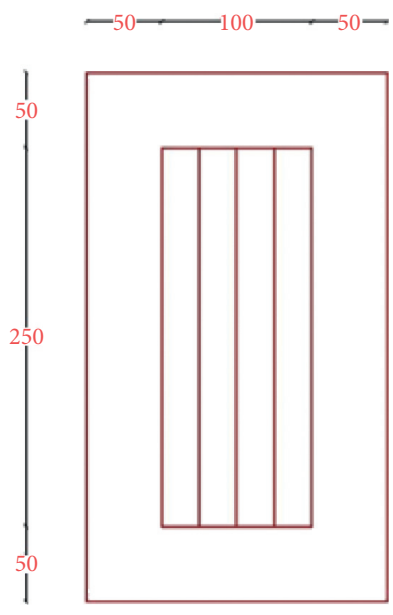

(a)

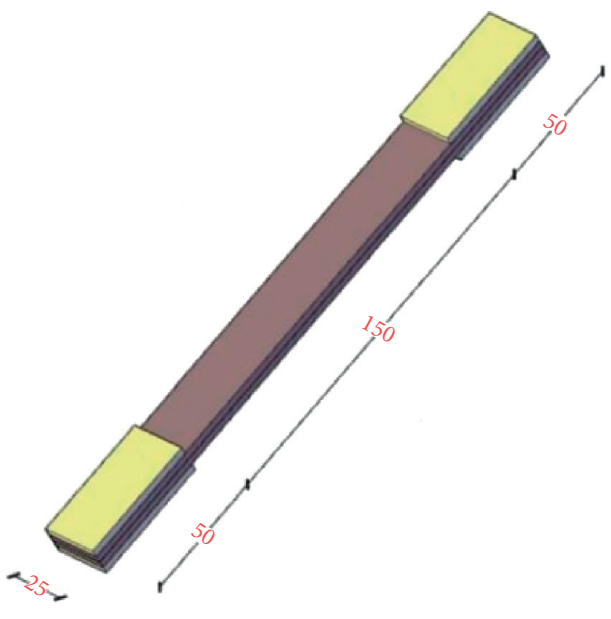

(b)

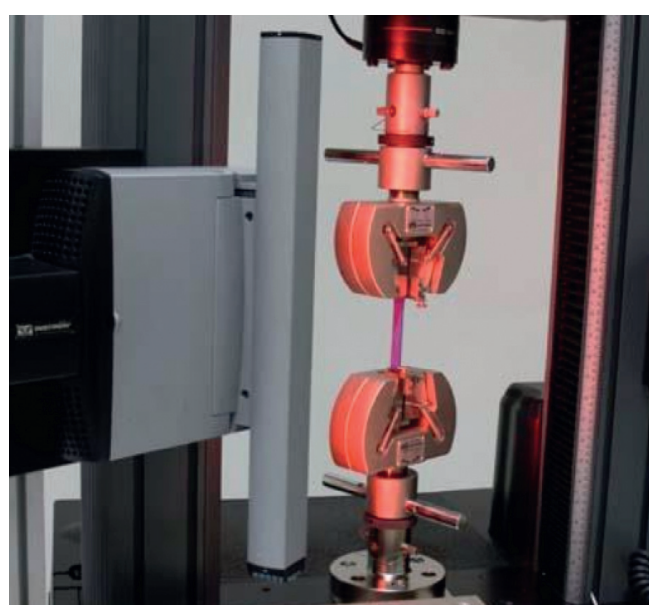

(c)

FIGURE 1: Dimensions of composite plates and specimens tested in direct longitudinal tension: (a) composite plate dimensions, (b) sample test dimensions, and (c) universal testing machine with the noncontact advanced video extensometer.

UD 200) used in this study were produced by Groupe Depestele, a French natural textile company. The 30\% biosourced DGEBA resin (CHS-EPOXY G520) was supplied by Spolchemie, a Czech chemical company known for its environmentally friendly products. The hardener is a $100 \%$ biosourced amine derived from cardanol (Phenalkamine NX 5619). Its amino hydrogen equivalent weight is $104 \mathrm{~g} \mathrm{eq}^{-1}$. The epoxy resin was mixed with the hardener using a highspeed mixer and considering a stoichiometric ratio of amino hydrogen to epoxy, a/e, equal to 1 . The composite plates were cured in laboratory conditions $\left\{20^{\circ} \mathrm{C} / 35-50 \% \mathrm{RH}\right\}$ for 3 weeks until stabilization of the polymerization process. This initial state corresponds to the starting time $T 0$ of the durability test program. The FFRP laminates had a fiber volume fraction around $16 \%$. Their glass transition temperature after initial cure (at $\mathrm{T0}$ ) was measured by differential scanning calorimetry (DSC) at a heating rate of $10^{\circ} \mathrm{C} / \mathrm{min}$ and was equal to $54^{\circ} \mathrm{C}$.

All the FFRP composite plates were then divided into six series that were placed in various environments, corresponding to different accelerated ageing conditions (see Table 1). The specimens were stored (or immersed in the case of an environment at $100 \% \mathrm{RH}$ ) vertically in the climatic/ bath chambers to favor an optimal 1D moisture diffusion across the thickness (see Figures 2(a) and 2(b)). In order to be uniform between figures, each ageing condition is associated to a specific color (e.g., $V 1$ is associated to red) and each time measurement is associated to a specific mark ("+" for 3 months, "•" for 6 months, "⿴囗 for 12 months, and "A" for 24 months).

Mechanical tests have been completed at $T 0$ and after exposure times of $3,6,12$, and 24 months. The tests were performed after shorter intervals at the beginning of the tests and then become rarer since a slight decrease of the degradation speed was expected and thus less information is necessary. Indeed, water sorption phenomena have a fast initial kinetics which slows down quite rapidly after a few weeks/months. We therefore expected to see a marked influence of RH in the short term (accelerated by T), and a slower evolution thereafter. This assumption has been validated by our experimental observations.

2.2. Testing Procedure. This study was part of a research project which involved a comprehensive durability test program on FFRP strengthening systems. This program consisted in performing various types of mechanical tests, to follow the evolutions of several performance indicators of these systems (tensile properties and interlaminar shear strength of the FFRP laminates and bond strength of the FFRP/concrete interface) versus exposure time in various accelerated ageing environments. The present paper is only focused on the evolution of the tensile capacity of the FRRP composite over ageing time.

As mentioned in the previous section, UD FFRP plates with the dimension of width $200 \times$ height $350 \mathrm{~mm}$ with two plies have been made by hand layup process. Samples with the dimension of width $25 \times$ height $250 \mathrm{~mm}$ (Figure 1(b)) have been cut from the plate using a water-cooled table diamond saw in order to ensure their integrity (which has been optically checked ex-post). In order to minimize the edge effects due to impregnation flaws, a strip of $5 \mathrm{~cm}$ wide of material is removed all around the perimeter of the plates before to cut the samples (Figure 1(a)). Glass fiber composite tabs are glued to each extremity of the samples using an epoxy adhesive, to improve the grip during tensile tests (Figure 1(b)). Direct longitudinal tensile tests were carried out on samples of the UD FFRP composite laminate (in the fiber direction) according to NF EN ISO 527-5 standard and French AFGC guidelines [21]. An Instron 5969 universal testing machine, equipped with a noncontact advanced video extensometer, was used to apply the loading speed of $1 \mathrm{~mm} / \mathrm{min}$ as advised in the standard method.

An actual tensile strength can be determined directly from the force monitored at failure and from the sample's cross-sectional area (width $\times$ thickness). However, this latter 
TABLe 1: Ageing conditions.

\begin{tabular}{lccr}
\hline Name & Temperature $\left({ }^{\circ} \mathrm{C}\right)$ & Humidity & Test schedule \\
\hline$V 1$ & 20 & $50 \% \mathrm{RH}$ (climatic chamber) & $3,6,12$, and 24 months \\
$V 2$ & 20 & $100 \%$ (immersion in water) & $3,6,12$, and 24 months \\
$V 3$ & 60 & $50 \% \mathrm{RH}$ (climatic chamber) & $3,6,12$, and 24 months \\
$V 4$ & 40 & $100 \%$ (immersion in water) & $3,6,12$, and 24 months \\
$V 5$ & 60 & $75 \% \mathrm{RH}$ (climatic chamber) & $3,6,12$, and 24 months \\
$V 6$ & 60 & $100 \%$ (immersion in water) & $3,6,12$, and 24 months \\
\hline
\end{tabular}

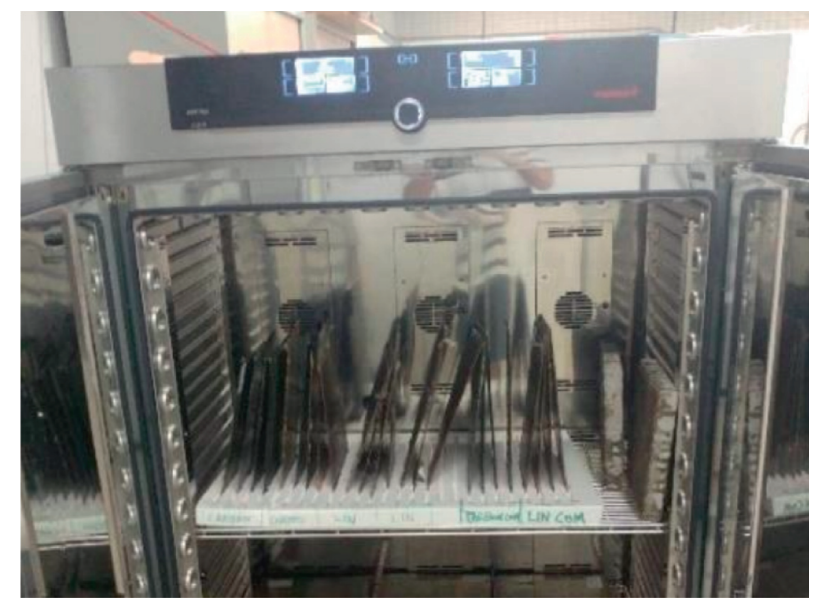

(a)

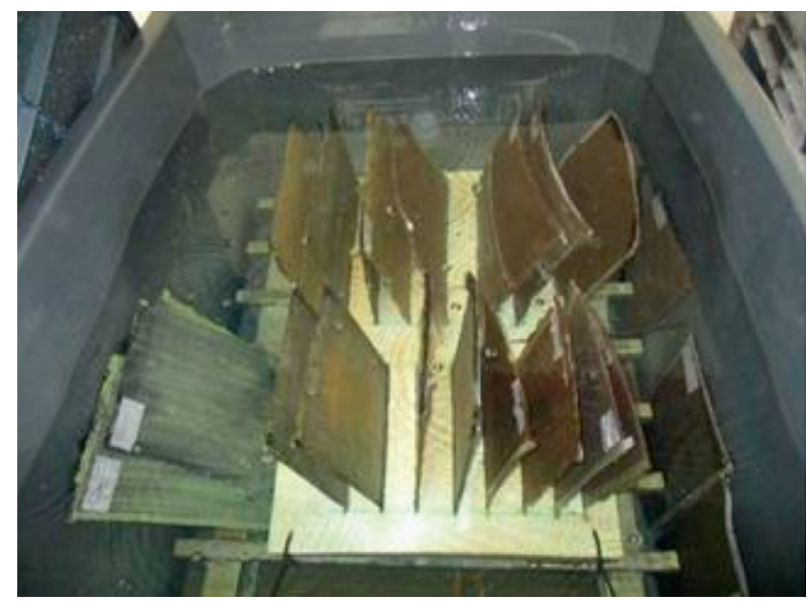

(b)

FIGURE 2: FFRP composite plates were stored vertically in the climatic/bath chambers: (a) climatic chamber for nonimmersed (RH $=50 \%$ or $75 \%)$ conditions; (b) bath chamber for immersed $(\mathrm{RH}=100 \%)$ conditions.

dimension is known to be not suitable for the design of structural reinforcement, since (i) the thickness of samples made by the hand layup process can vary within the plate and (ii) it is essentially the active section of fibers which provides its tensile strength to the sample. Thus, as prescribed in ASTM D7565/D7565M-10 (2017) [22], the tensile capacity per unit width (for a given number of layers) of the composites, as defined by the following equation, is monitored:

$$
f_{\text {fu }}=\frac{\text { failure force }}{\text { sample's width } \times \text { number of layers }} \text {. }
$$

Aged specimens were tested 2 to 3 days after their removal from the climatic/bath chambers. This time corresponds to the time necessary for the preparation of the samples before the test (cutting of test samples, preparation of the grip tabs, and gluing of the gauges). The number of samples tested for each type of condition and for each term of the test program is shown in Table 2. In the following Sections 3 and 4, statistical processing of data will be carried out. Relevancy of such statistical processing is debatable with regard to the limited number of samples available at each time measurement and for each ageing condition (from 3 to 5 samples). At most, the calculation of the mean value makes sense, whilst that of variance has less meaning. However, the statistical processing that will be carried out in our study has the objective to estimate parameters of a degradation model (a TED process) on the whole (at different times). This estimation is based on the maximization of the likelihood function (see equation (14) in Section 3.4), and this latter function aggregates, for each ageing condition, all the data available at time $T 0,3,6,12$, and 24 months. It means that the statistical processing relies actually, for instance, for $V 1$ ageing condition, on 47 samples or data $(47=33$ at $T 0+4$ at 3 months +3 at 6 months +4 at 12 months +3 at 24 months). The total number of data available for the parameters' estimation of the stochastic TED process model is given in the last column of Table 2.

Figures 3(a)-3(f) show examples of typical tensile force (per unit width) vs. strain curves for the six different ageing conditions at initial time $T 0$ and after 3 and 24 months. The tensile capacity used later in the model corresponds to the value of tensile force reached at the failure of the sample.

\section{Probabilistic Analysis of Accelerated Degradation Processes}

3.1. Tweedie Exponential Dispersion Model. A stochastic process is called an exponential dispersion (ED) degradation process $\{Y(t), t \geq 0\}$ if satisfying the following properties: 
TABLe 2: Number of tests/data.

\begin{tabular}{lcccccc}
\hline Type & Time $=$ T0 & 3 months & 6 months & 12 months & 24 months & Total number of data \\
\hline$V 1$ & & 3 & 4 & 4 & 3 & 47 \\
$V 2$ & 4 & 3 & 3 & 4 & 3 & 46 \\
$V 3$ & 33 & 4 & 4 & 3 & 3 & 47 \\
$V 4$ & & 4 & 4 & 3 & 48 \\
$V 5$ & 3 & 5 & 5 & 3 & 49 \\
$V 6$ & & 4 & & & \\
\hline
\end{tabular}

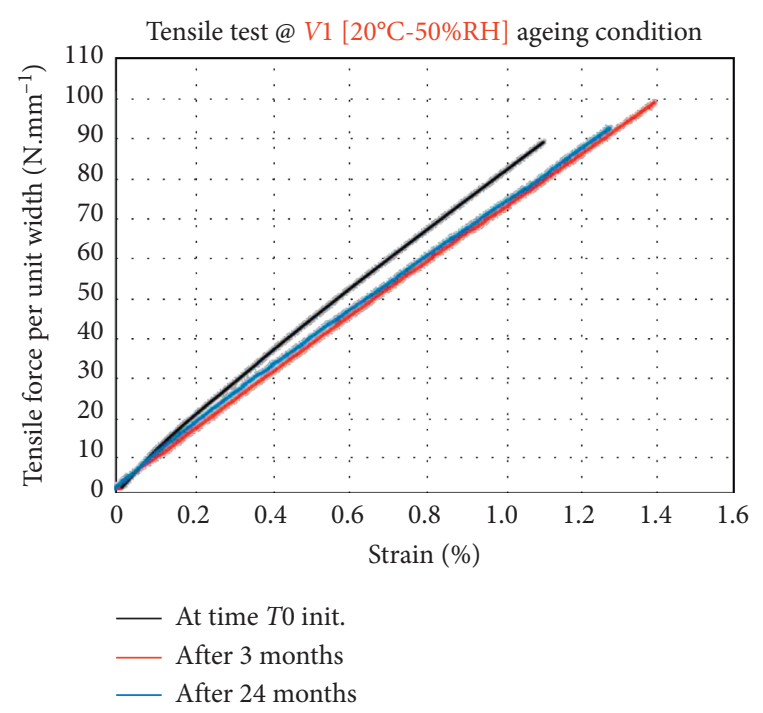

(a)

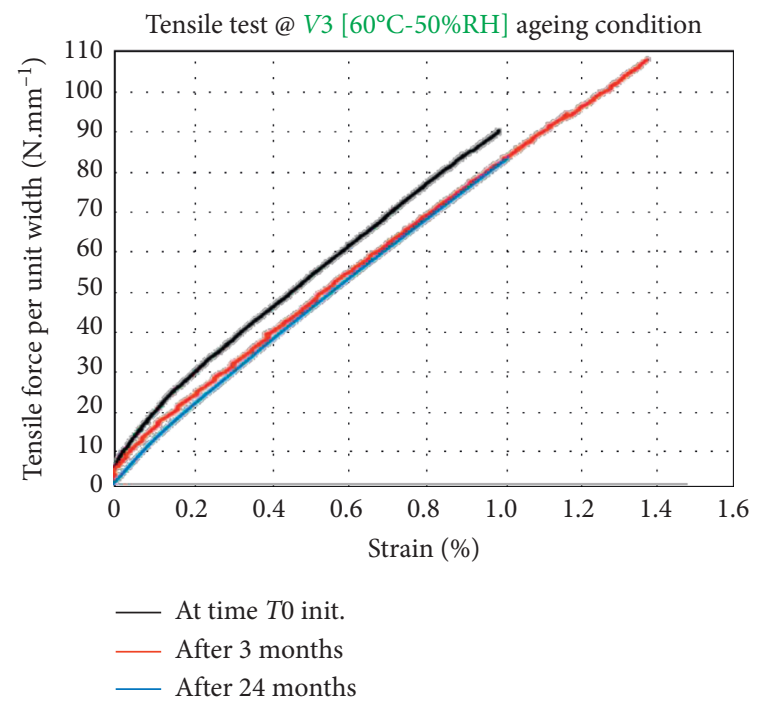

Tensile test @V2 $\left[20^{\circ} \mathrm{C}-100 \% \mathrm{RH}\right]$ ageing condition

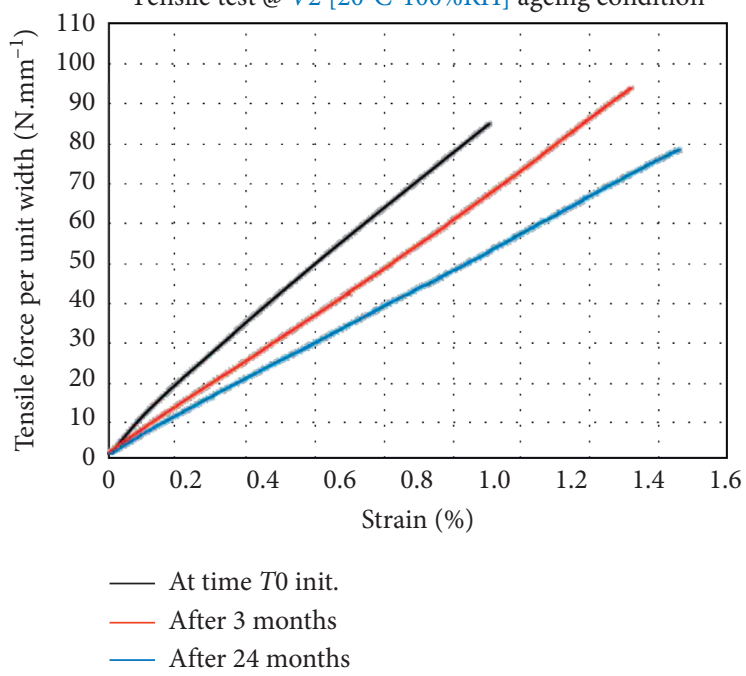

(b)

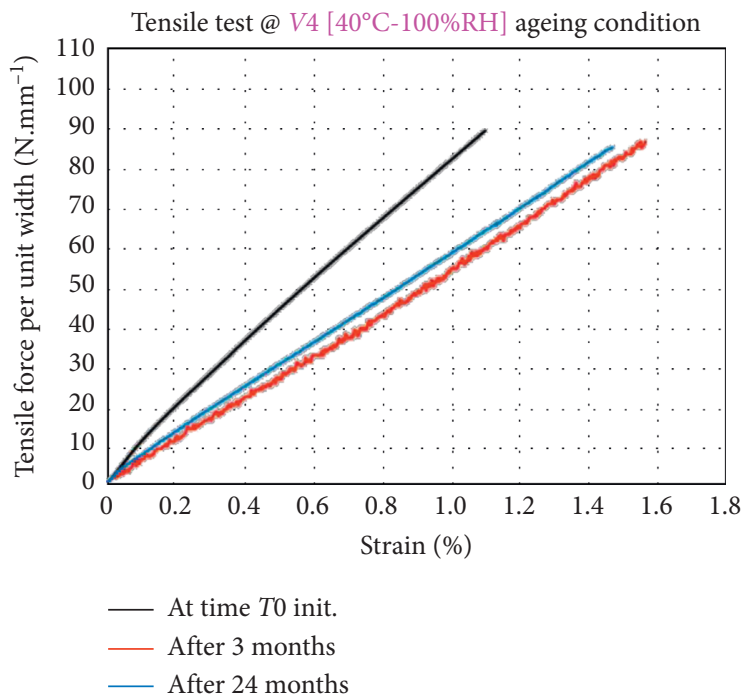

(c)

(d)

FIgURE 3: Continued. 


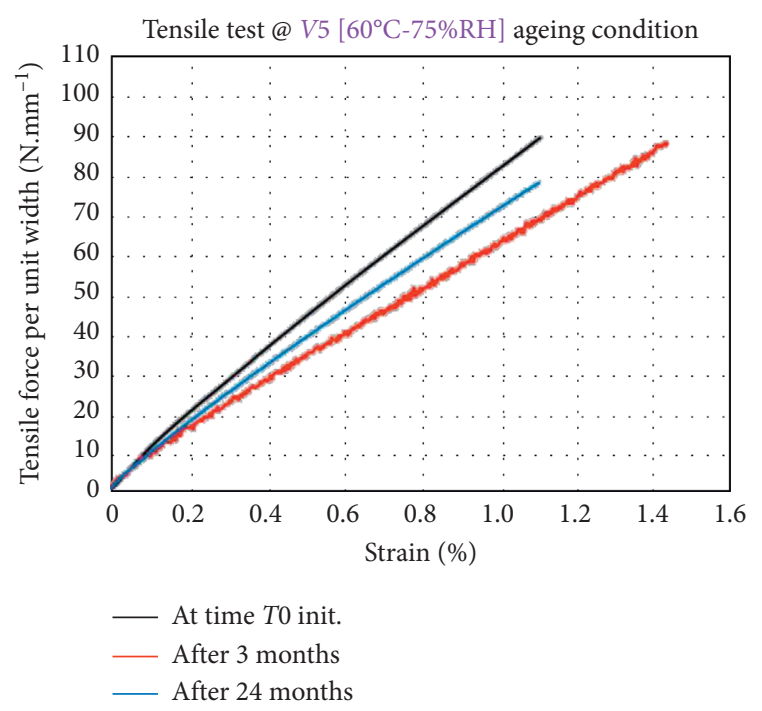

(e)

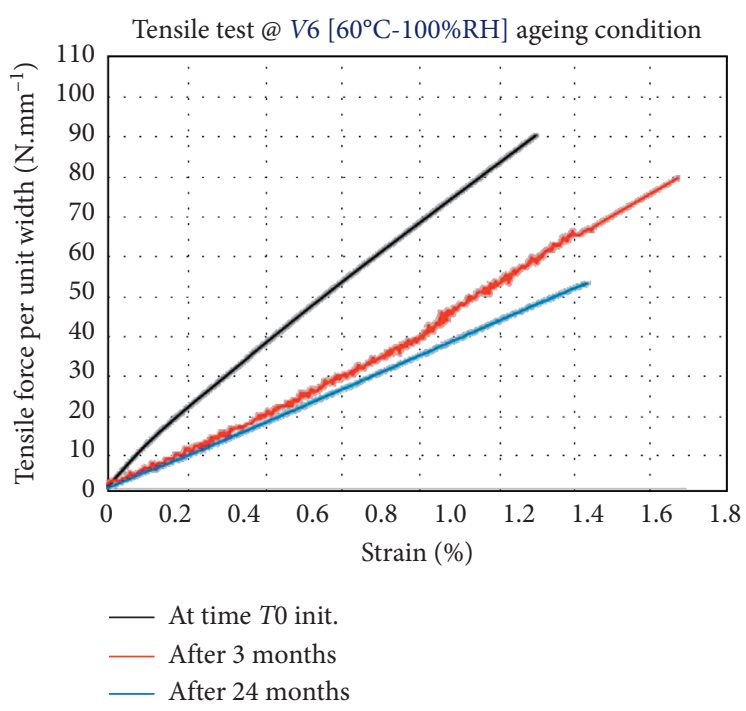

(f)

Figure 3: Typical tensile test curves for the different ageing conditions and at varying test times (at initial time T0, after 3 months and after 24 months): (a) $V 1\left(20^{\circ} \mathrm{C}-50 \% \mathrm{RH}\right)$ ageing condition, (b) $V 2\left(20^{\circ} \mathrm{C}-100 \% \mathrm{RH}\right)$ ageing condition, (c) $V 3\left(60^{\circ} \mathrm{C}-50 \% \mathrm{RH}\right)$ ageing condition, $(\mathrm{d})$ $V 4\left(40^{\circ} \mathrm{C}-100 \% \mathrm{RH}\right)$ ageing condition, (e) $V 5\left(60^{\circ} \mathrm{C}-75 \% \mathrm{RH}\right)$ ageing condition, and (f) $V 6\left(60^{\circ} \mathrm{C}-100 \% \mathrm{RH}\right)$ ageing condition.

(1) $Y(0)=0$ with probability one

(2) $\{Y(t), t \geq 0\}$ has independent increments on nonoverlapping intervals; that is, $Y\left(t_{2}\right)-Y\left(t_{1}\right)$ and $Y\left(t_{4}\right)-Y\left(t_{3}\right)$ are independent for $0 \leq t_{1} \leq t_{2} \leq t_{3} \leq t_{4}$

(3) Each increment follows an ED distribution, i.e.,

$$
\begin{aligned}
Y(t+\Delta t)-Y(t) \sim & \operatorname{ED}(\mu(\Lambda(t+\Delta t ; q) \\
& -\Lambda(t ; q)), \lambda), \quad \text { for } \forall \Delta t>0,
\end{aligned}
$$

where the probability density function (PDF) of ED distribution $\operatorname{ED}(\mu \Lambda(t ; q), \lambda)$ is

$$
f_{Y}(y, t \mid \mu, q, \lambda)=c(\lambda, y, t) \exp \{\lambda[y \theta-\Lambda(t ; q) \kappa(\theta)]\} .
$$

(i) $c(\cdot)$ is a canonical function guaranteeing that the cumulative distribution function (CDF) of equation (3) is normalized and no more than one.

(ii) $\lambda$ is called the index or diffusion parameter.

(iii) $\kappa(\cdot)$ is called the cumulant function of ED distribution since its first derivative $\kappa^{\prime}(\cdot)$ gives the successive cumulants of this distribution.

(iv) $\mu$ is called the mean value parameter and is implicitly found in equation (3) since it satisfies $\mu=\kappa^{\prime}(\theta) \triangleq \tau(\theta)$.

(v) $\Lambda(t ; q)$ is the function of time $t$, which is specified according to degradation physics or empirical observations and $\mu \Lambda(t ; q)$ can be regarded as the mean degradation path. Function $\Lambda(t ; q)$ is generally nonlinear and can take into account a phase shift in time of the observed phenomenon, $b$. A typical flexible form is that of power function where $\Lambda(t ; q)=(t-b)^{q}$ for $t \geq b$. If $q=1$, then $\Lambda(t ; q)=$ $(t-b)$, and the degradation path is linear after $t=b$.
Finally, the mean degradation path can use the following form to take into account an initial value $\xi$ of the degradation:

$$
\mu \Lambda(t ; q)=\mu(t-b)^{q}+\xi
$$

The mean of $\mathrm{ED}$ process is $E(Y(t))=\mu \Lambda(t ; q)$, as mentioned above. The variance is $\operatorname{Var}(Y(t))=V(\mu) \Lambda$ $(t ; q) / \lambda$, where $V(\mu)=\kappa^{\prime \prime}\left(\tau^{-1}(\mu)\right)$ is the unit variance function and $\kappa^{\prime \prime}(\theta)$ is the second derivative of $\kappa(\theta)$ with respect to $\theta$.

The Tweedie exponential dispersion (TED) model is an important special class of ED models where

$$
V(\mu)=\mu^{p}, \quad p \in(-\infty, 0] \cup[1, \infty) .
$$

The TED process includes Wiener process (for $p=0$ ), Gamma process $(p=2)$, and IG process $(p=3)$ models.

The function $\kappa(\theta)$ can be obtained for the TED model by solving the equations $\kappa^{\prime}\left(\tau^{-1}(\mu)\right)=\left.(\mathrm{d} \mu / \mathrm{d} \theta)\right|_{\theta=\tau^{-1}(\mu)}=\mu^{p}$, and the solution can be expressed as

$$
\kappa(\theta)= \begin{cases}\exp (\theta), & p=1, \\ -\ln (-\theta), & p=2, \\ \frac{[(1-p) \theta]^{((p-2) /(p-1))}}{2-p}, & p \neq 1,2 .\end{cases}
$$

Although $\kappa(\theta)$ has an analytic form, it remains that $c(\cdot)$ in equation (3) has no closed form except for some special values. An approximated method, the saddle-point approximation method (SAM), can be used to obtain the PDF of TED. According to the previous research $[23,24]$, the SAM provides a highly accurate approximation for the TED 
model, which is expressed in terms of the unit deviance $d(y, t \mid \mu, q)$ :

$$
f(y, t \mid \mu, q, \lambda) \cong \sqrt{\frac{\lambda}{2 \pi \Lambda^{1-p}(t ; q) y^{p}}} \cdot \exp \left(-\frac{\lambda \Lambda(t ; q)}{2} d(y, t \mid \mu, q)\right),
$$

where this unit deviance takes the following forms, respectively, for the three cases $p=1, p=2$, or $p \neq 1,2$ :

$$
d(y, t \mid \mu, q, \lambda)= \begin{cases}2\left\{\frac{y}{\Lambda(t ; q)} \ln \frac{y}{\mu \Lambda(t ; q)}-\left(\frac{y}{\Lambda(t ; q)}-\mu\right)\right\}, & p=1 \\ 2\left\{\ln \frac{\mu \Lambda(t ; q)}{y}+\frac{y}{\mu \Lambda(t ; q)}-1\right\}, & p=2, \\ 2\left\{\frac{y^{2-p} \Lambda^{p-2}(t ; q)}{(1-p)(2-p)}-\frac{y \mu^{1-p}}{(1-p) \Lambda(t ; q)}+\frac{\mu^{2-p}}{2-p}\right\}, & p \neq 1,2 .\end{cases}
$$

The two cases with $p$ equal to 1 and 2, which correspond, respectively, to the Poisson and Gamma process, have specific PDF expressions. As we aim developing a more generic approach, we will study the expression of the unit deviance when $p \neq 1,2$.

3.2. Reliability Function. According to the definition of soft failure, when the amount of degradation reaches a prespecified critical level $\omega$, failure occurs, that is, the product lifetime $T=\inf \{t: Y(t)=\omega\}$. The CDF of lifetime distribution is $F(t)=P(T \leq t)$, and the reliability function is $R(t)=1-F(t)$.

Generally speaking, it is not easy to have analytical expressions of $F(t)$ and $R(t)$. An approximation method can be used to compute the $\mathrm{CDF}$ and reliability function. According to the results obtained by Jørgensen [25] and Hong and Ye [26], when $\lambda \Lambda(t ; q)$ is large, which is often true when $t$ is large, the lifetime $T$ is approximately normal with mean $\mu \Lambda(t ; q)$ and variance $V(\mu) \Lambda(t ; q) / \lambda$. Hence, the reliability function and PDF of the lifetime distribution for the TED model can be approximated as

$$
\left\{\begin{array}{l}
R(t \mid \mu, \lambda) \cong 1-\Phi\left(\sqrt{\frac{\lambda}{\mu^{p}}}\left(\mu \sqrt{\Lambda(t ; q)}-\frac{\omega}{\sqrt{\Lambda(t ; q)}}\right)\right) \\
f(t \mid \mu, \lambda) \cong \frac{\Lambda^{\prime}(t ; q)}{2} \sqrt{\frac{\lambda}{\mu^{p} \Lambda(t ; q)}}\left(\mu+\frac{\omega}{\Lambda(t ; q)}\right) \phi\left(\sqrt{\frac{\lambda}{\mu^{p}}}\left(\mu \sqrt{\Lambda(t ; q)}-\frac{\omega}{\sqrt{\Lambda(t ; q)}}\right)\right)
\end{array}\right.
$$

where $\Phi(\cdot)$ and $\phi(\cdot)$ are the CDF and PDF of the standard normal distribution, respectively. It is noted that some characteristic functions (e.g., $u^{\text {th }}$-quantile life and mean time to failure (MTTF)) have closed-form expressions according to this approximation approach.

Denoting the standard normal quantile $\Phi^{-1}(u)$ as $z_{u}$, an approximation of the $u^{\text {th }}$ lifetime quantile is

$$
t_{u} \cong\left[\frac{\left(z_{u} \sqrt{\left(\mu^{p} \Lambda(t ; q) / \lambda\right)}+\sqrt{z_{u}^{2}\left(\mu^{p} \Lambda(t ; q) / \lambda\right)+4 \mu \Lambda(t ; q) \omega}\right)^{2}}{4 \mu^{2} \Lambda^{2}(t ; q)}\right]^{1 / q} .
$$

And, thus, the mean time to failure (MTTF) is approximated as

$$
\operatorname{MTTF} \cong\left[\frac{\omega}{\mu \Lambda(t ; q)}\right]^{1 / q} .
$$

3.3. Acceleration Test. Temperature and humidity are the two stress variables in the accelerated life test. The generalized Eyring model (GEM) [27] describes the relationship between life and temperature and humidity. In the TED model, $\mu$ denotes the degradation rate which clearly should 
change when the acceleration stress is different. Therefore, the parameter $\mu$ is assumed to be affected by the stress's combination $(T, \mathrm{RH})$ and can be written as

$$
\mu(T, \mathrm{RH})=a \exp \left(-\frac{c_{1}}{T}+c_{2} \cdot \mathrm{RH}+\frac{c_{3} \cdot \mathrm{RH}}{T}\right),
$$

where $T$ is the thermodynamic temperature in kelvin (K) and $\mathrm{RH}$ is the relative humidity (\%). The parameters $a, c_{1}, c_{2}$, and $c_{3}$ need to be estimated.

To use ED process for the purposes mentioned above, initial symbols and assumptions have to be put forward:

(1) The number of stress $\left(s_{k}\right)$ combination levels is denoted by " $d$ ", and, thus, $s_{k}, k=1,2, \ldots, d$. In our test, $d=6$. The test is destructive (whereas works found in the literature mainly deal with nondestructive tests or continuous degradation monitoring); that is, a unit can only be detected once; that has to be considered in the writing of the likelihood function (equation (14), later). Each stress combination level $\left(T_{k}\right.$ and $\mathrm{RH}_{k}$, where $\left.k=1,2, \ldots, d\right)$ is loaded following the constant stress loading scheme. $T_{k}$ and $\mathrm{RH}_{k}$ represent the $k^{\text {th }}$ stresses combination.

(2) The total number of units available for the test is $N$; $N_{k}$ of them are allocated to the stress combination level $s_{k}$, such that $\sum_{k=1}^{d} N_{k}=N$.

(3) Let $m_{k}$ and $t_{k j}$, respectively, represent the number and $j^{t h}$ detection time for units at the stress level $s_{k}$. The corresponding number of samples is $n_{k j}$, such that $\sum_{j=1}^{m_{k}} n_{k j}=N_{k}$.

(4) Under each stress level $s_{k}, k=1, \ldots, d$, the degradation characteristic $y_{k j i}$ of the $i^{\text {th }}$ unit $\left(i=1, \ldots, n_{k j}\right)$ follows ED distribution with mean $\mu(T, \mathrm{RH}) \cdot \Lambda(t)$ and diffusion parameter $\lambda$. It is assumed that the parameter $\lambda$ is a constant over stresses, which agrees with our daily observation of the product's physical characteristic.

(5) The degradation of mechanical performance is divided into two stages. The first stage increases due to the postcuring process, which physically corresponds to the continuation of residual monomer reticulation during ageing, leading to the increase of cross-linking degree of the epoxy network. Then, in the second stage, the mechanical performance decreases down to failure. Under the stress combination level $s_{k}$, the final value of mechanical performance in postcuring process is $\xi_{k}, k=$
$1,2, \ldots, d$, which is also the initial value of the second stage, and which is different under different stress combination levels. In engineering, we are more concerned about the mechanical properties in the second stage. Therefore, only the second stage for degradation modeling will be considered.

At this stage, the mechanical performance $y$ can be expressed as

$$
y_{k}=\xi_{k}-x\left(t ; T_{k}, \mathrm{RH}_{k}\right)
$$

where $x\left(t ; T_{k}, \mathrm{RH}_{k}\right)$ follows TED process with an initial value taken at zero for $t=0$.

(6) A unit is assumed to fail at a time $T$ when its degradation $Y(t)$ crosses a predetermined failure threshold $\omega$. It is assumed that the function $\Lambda(t ; q)=\left(t-b_{k}\right)^{q}, k=1,2, \ldots, d . b_{k}$ is the shift of time affected by the postcuring effects.

(7) The unknown parameters to determined are $\Xi=\left(\xi_{1}, \xi_{2}, \ldots, \xi_{d}, b_{1}, b_{2}, \ldots, b_{d}, a, c_{1}, c_{2}, c_{3}, p, q, \lambda\right)$.

3.4. Parameter Estimation. It aims at deriving process parameters with explicit expressions. The unknown parameters of the TED process have to be estimated from the data provided by actual (experimental) accelerated destructive tests. Following the argument in the assumptions, the PDF $f\left(\xi_{k}-y_{k j i}\right)$ of the degradation data $y_{k j i}$ is TED distribution with mean $a \Lambda(t) \exp \left(-\left(c_{1} / T\right)+c_{2} \cdot \mathrm{RH}+\left(\left(c_{3} \cdot \mathrm{RH}\right) / T\right)\right)$ and diffusion parameter $\lambda$. Therefore, the likelihood function of the proposed model is

$$
L=\prod_{k=1}^{d} \prod_{j=1}^{m_{k}} \prod_{i=1}^{n_{k j}} f\left(\xi_{k}-y_{k j i}\right) .
$$

In the above equation, each sample, corresponding to subscript $k j i$ ( $i^{\text {th }}$ sample, measured at the $j^{\text {th }}$ time, under the $k^{\text {th }}$ stress combination), is counted once since the tests are destructive.

Then, the log-likelihood function of $\Xi$ up to a constant is

$$
\begin{aligned}
l= & \frac{N}{2} \ln (\lambda)-\frac{1}{2} \sum_{k=1}^{d} \sum_{j=1}^{m_{k}} \sum_{i=1}^{n_{k j}}\left[(1-p) \ln \Lambda\left(t_{k j} ; q\right)\right. \\
& \left.+p \ln \left(\xi_{k}-y_{k j i}\right)+\lambda \Lambda\left(t_{k j} ; q\right) d\left(y_{k j i} ; \mu_{i}\right)\right],
\end{aligned}
$$

where 


$$
d\left(y_{k j i} ; \mu_{i}\right)= \begin{cases}2\left\{\frac{\xi_{k}-y_{k j i}}{\Lambda\left(t_{k j} ; q\right)} \ln \frac{\xi_{k}-y_{k j i}}{\mu_{i} \Lambda\left(t_{k j} ; q\right)}-\left(\frac{\xi_{k}-y_{k j i}}{\Lambda\left(t_{k j} ; q\right)}-\mu_{i}\right)\right\}, & p=1, \\ 2\left\{\ln \frac{\mu_{i} \Lambda\left(t_{k j} ; q\right)}{\xi_{k}-y_{k j i}}+\frac{\xi_{k}-y_{k j i}}{\mu_{i} \Lambda\left(t_{k j} ; q\right)}-1\right\}, & p=2, \\ 2\left\{\frac{\left(\xi_{k}-y_{k j i}\right)^{2-p} \Lambda^{p-2}\left(t_{k j} ; q\right)}{(1-p)(2-p)}-\frac{\left(\xi_{k}-y_{k j i}\right) \mu^{1-p}}{(1-p) \Lambda\left(t_{k j} ; q\right)}+\frac{\mu_{i}^{2-p}}{2-p}\right\}, & p \neq 1,2 .\end{cases}
$$

Taking the first-order derivation of $l(\Xi \mid y)$ to respect $\xi_{1}, \xi_{2}, \ldots, \xi_{d}$ and $\lambda$, then we can have

$$
\frac{\partial l}{\partial \lambda}=\frac{N}{2 \lambda}-\frac{1}{2} \sum_{k=1}^{d} \sum_{j=1}^{m_{k}} \sum_{i=1}^{n_{k j}}\left[\Lambda\left(t_{k j} ; q\right) d\left(y_{k j i} ; \mu_{i}\right)\right] .
$$

Setting this equation to zero leads to

$$
\hat{\lambda}=\frac{N}{\sum_{k=1}^{d} \sum_{j=1}^{m_{k}} \sum_{i=1}^{n_{k j}}\left[\Lambda\left(t_{k j} ; q\right) d\left(y_{k j i} ; \mu_{i}\right)\right]} .
$$

Substituting (18) into (15), we have a profile log-likelihood function $l\left(\xi_{1}, \xi_{2}, \ldots, \xi_{d}, a, c_{1}, c_{2}, c_{3}, p, q\right)$. The estimators of $\xi_{1}, \xi_{2}, \ldots, \xi_{d}, b_{1}, b_{2}, \ldots, b_{d}, a, c_{1}, c_{2}, c_{3}, p$ and $q$ can be obtained as follows:

$$
\begin{aligned}
& \left(\widehat{\xi}_{1}, \widehat{\xi}_{2}, \ldots, \widehat{\xi}_{d}, \widehat{b}_{1}, \widehat{b}_{2}, \ldots, \widehat{b}_{d}, \widehat{a}, \widehat{c}_{1}, \widehat{c}_{2}, \widehat{c}_{3}, \widehat{p}, \widehat{q}\right) \\
& \quad=\underset{\xi_{k} \in \Omega_{\xi}, \ldots p \in \Omega_{p}, q \in \Omega_{q}}{\operatorname{Argmax}} l(\Xi) .
\end{aligned}
$$

Substituting $\quad\left(\widehat{\xi}_{1}, \widehat{\xi}_{2}, \ldots, \widehat{\xi}_{d}, \widehat{b}_{1}, \widehat{b}_{2}, \ldots, \widehat{b}_{d}, \widehat{a}, \widehat{c}_{1}, \widehat{c}_{2}, \widehat{c}_{3}\right.$, $\widehat{p}, \widehat{q})$ into (15), then the MLEs $\hat{\lambda}$ can be obtained. These estimated parameters are denoted as $\widehat{\Xi}$. The estimate of the reliability function can be obtained by substituting the estimated parameters $\widehat{\Xi}$ into the reliability functions in equation (9).

\section{Lifetime Prediction}

4.1. Experimental Results. Mechanical tensile tests were first carried out on unaged specimens, providing the initial reference performance before ageing. Samples were then tested after 3, 6, 12, and 24 months ageing in various conditions. The experimental results, i.e., the values of the tensile capacity of the FFRP laminates versus ageing time in various accelerated environments, are shown in Figures 4(a) and 4(b). To analyze the degradation trend under different acceleration conditions, the mean degradation paths of tensile capacity per unit width are also shown in continuous straight lines connecting the averaged values at each time and for each ageing conditions. Some mean paths might appear to not connect the mean values, and it is only due to possible overlaying of dots from different ageing conditions.

Three typical profiles of degradation can be distinguished from the experimental observations (see Figures $4(\mathrm{c})$ ). The first profile is associated to a continuous decrease of the performance (profile I in Figure 4(c)). This profile is representative of the degradation under immersed $V 6$ ageing conditions (see Figures 4(b)). The second profile shows a peak performance followed by a delayed decrease of performance (profile II in Figure 4(c)). This profile can be considered as representative of the degradation under $V 2$, $V 3, V 4$, and $V 5$ ageing conditions (see Figures 4(a) and 4(b)). The third profile corresponds to an initial decrease of performance and a delayed portion of second profile, i.e., a decrease after an increase (profile III in Figure 4(c)). Performance degradation curve under $V 1$ ageing condition is representative of this profile (see Figure 4(a)).

Temperature and humidity have obvious influences on the postcuring effect. We must admit that we were surprised by the irregular fluctuations of the degradation paths in the postcuring stage. However, this phenomenon has been previously observed in the literature $[28,29]$ and is interpreted as the consequence of two antagonistic physical mechanisms (see Figure 4(d)). A first one ("ascending branch"), associated to the postcure of the resin (reaction of residual monomers), which results in an increase in performance over time. A second one ("descending branch") may be related to material and interface degradations or other phenomena decreasing performances such as resin plasticization.

After the postcuring stage, the tensile capacity per unit width will decrease, which is the gradual ageing stage. As the degradation process in the postcuring stage is irregular, only degradation analysis for the ageing stage is performed. Therefore, for the accelerated condition $V 1$, only the degradation data in the $12^{\text {th }}$ and $24^{\text {th }}$ months are used; for the accelerated condition $V 2$, only the degradation data in the $6^{\text {th }}, 12^{\text {th }}$, and $24^{\text {th }}$ months are used, and for the accelerated conditions $V 3, V 4, V 5$, and $V 6$, the degradation data in the $3^{\text {th }}, 6^{\text {th }}, 12^{\text {th }}$, and $24^{\text {th }}$ months are applied for the degradation analysis.

4.2. Estimation of TED Process Model Parameters. The approach detailed in Section 3 is applied to describe the degradation process is described by TED process. And considering that the power function is flexible, the mean degradation path is set as a power function; that is, $\mu_{k} \Lambda(t ; q)=\mu_{k}\left(t-b_{k}\right)^{q}+\xi_{k}, \quad k=1,2, \ldots, 6$ stands the 6 different accelerated conditions $V_{k}$. The initial value of the ageing stage is denoted as $\xi_{k}, k=1,2, \ldots, 6$ for the accelerated condition $V_{k}$. The parameter $\mu_{k}$ is affected by 


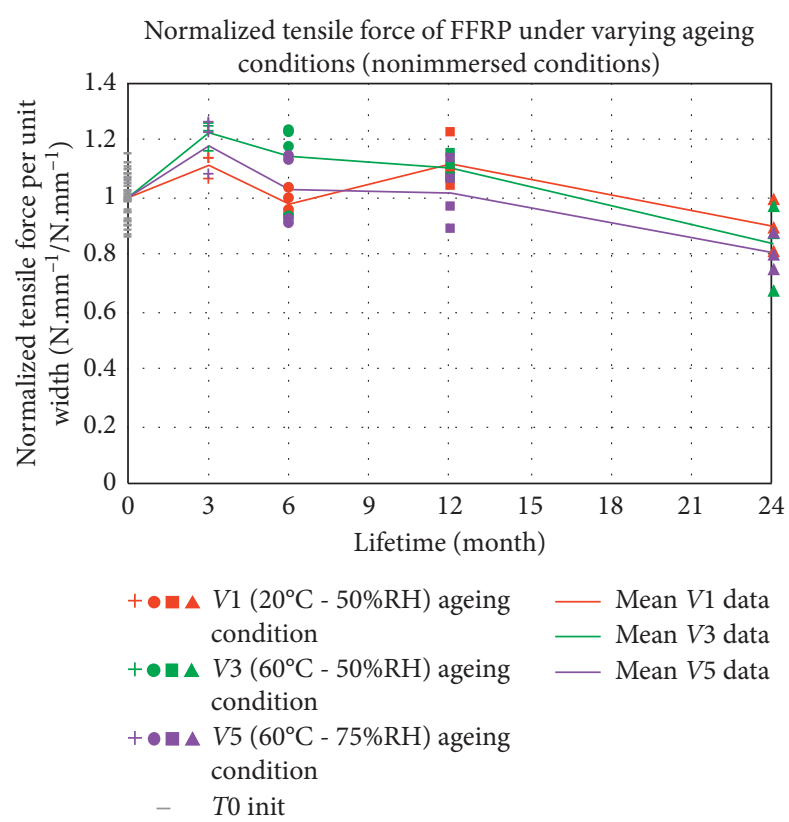

(a)

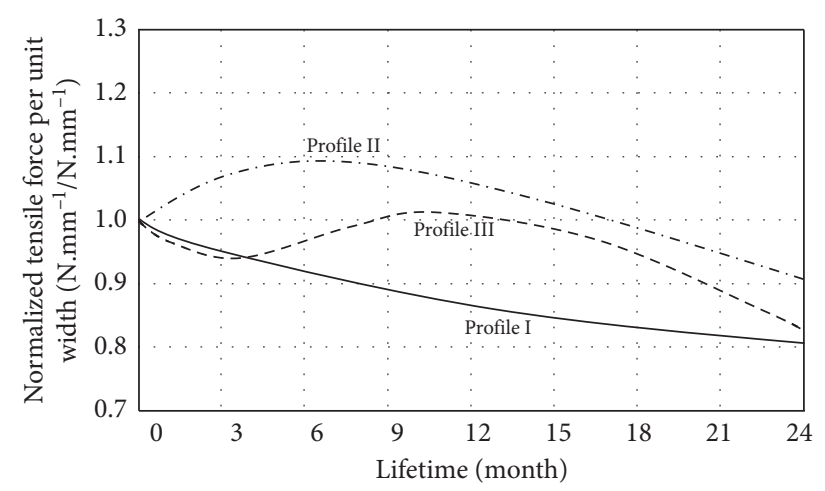

(c)

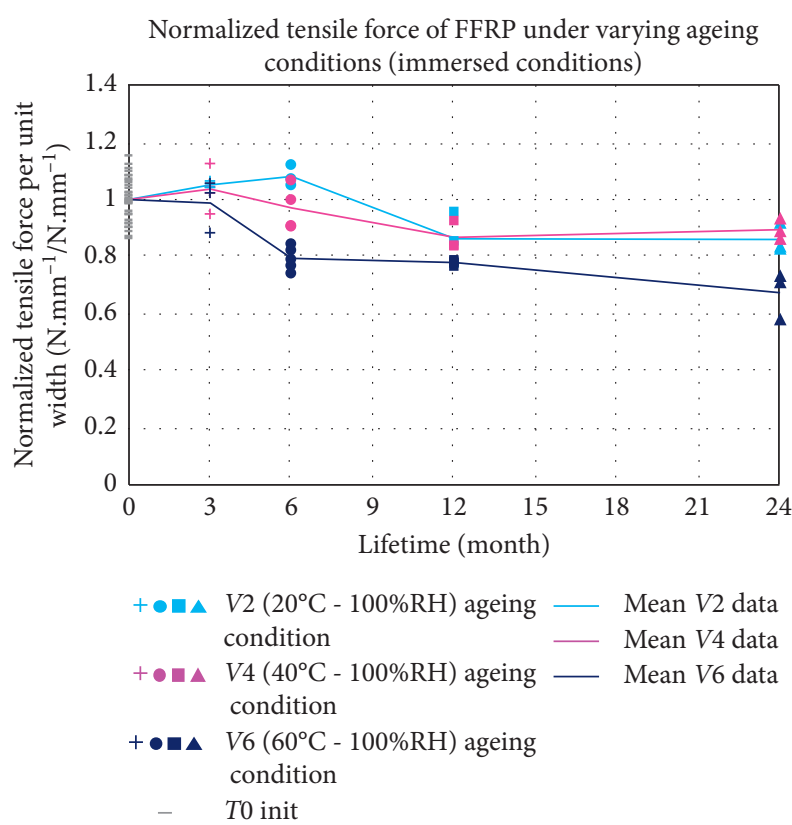

(b)

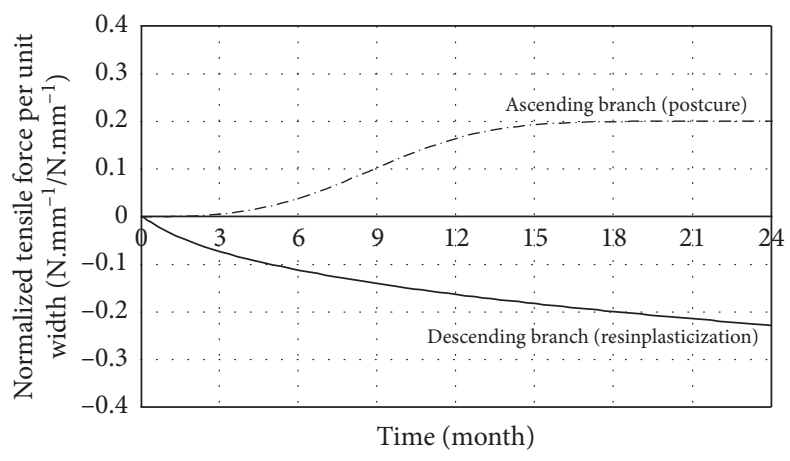

(d)

FIGURE 4: Results of tensile capacity per unit width value (normalized by the average of initial values): (a) for nonimmersed (RH $=50 \%$ or $75 \%)$ conditions, (b) for immersed $(\mathrm{RH}=100 \%)$ conditions, $(\mathrm{c})$ three typical profiles of the degradation curves, and (d) two antagonistic physical mechanisms.

temperature and humidity, and the relationship is described by GEM (in equation (12)). The MLE is used to obtain the estimates of the parameters, which are shown in Figure 5. The detail workflow of the estimation of the TED process parameters is shown in Figure 5. Figure 5 completes this Figure 5 with the estimated values of these parameters.

Finally, to show the fitting effect of the model on the data, the average degradation trajectory for fitting comparison is used, as shown in Figures 6(a)-6(f). The ED model performs well to describe the mean degradation paths at the six testing conditions. Furthermore, the SSE (the sum of squares due to error) at different stress levels is very small and ranges from $9.563 \times 10^{-4}$ (for V1) to $1.891 \times 10^{-2}$ (for V3). Therefore, it can be stated that the ED model is suitable to analyze the degradation path of tensile capacity per unit width. All SSE values are reported within Figures 6(a)-6(f).
4.3. Lifetime Estimation Based on Design Codes. When parameters of TED degradation process are estimated, it is thus possible to estimate the lifetime of FFRP laminates under a specific environmental condition of temperature and relative humidity. In the following, the condition $T=20^{\circ} \mathrm{C}$ and $\mathrm{RH}=50 \%$ are considered for reference. However, since the expression of the degradation rate $\mu$ as a function of $T$ and $\mathrm{RH}$ is embedded in the TED process set of parameters, any other environmental condition could be used.

Eventually, to estimate the lifetime, it is first necessary to state a criterion that should be considered to ensure a safe functioning of the FFRP system. In other words, the threshold value $\omega$ (found in equation (9)) defined as the maximum acceptable value of degradation of the mechanical performance is required. To find this threshold value, we rely on the existing design codes, i.e., ACI 440 2R-17 [30], TR55 


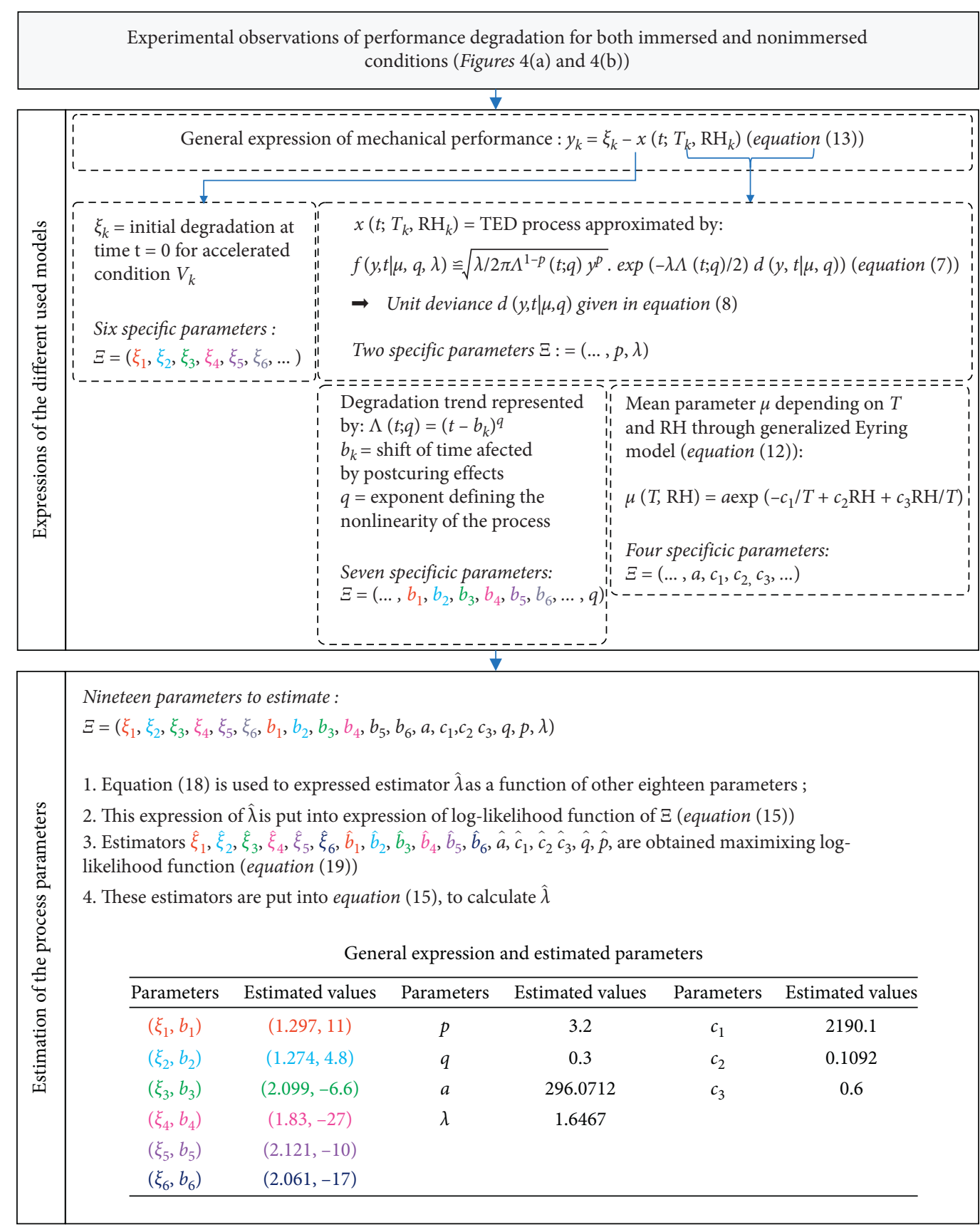

FIGURE 5: General expressions and workflow used to estimate the TED process parameters.

[31], FIB Bulletin 40 [32], and AFGC guide [21]. Even if our study concerns tensile capacity per unit width and not actually strength, the formalisms used for strength in the specific guidelines will be considered as reliable for capacity per unit width in the following.

For instance, in the case of ACI 440 2R-17 [30] recommendations, the design tensile strength (or capacity) at the ultimate limit state of FRP $f_{\text {fu }}$ is

$$
f_{\mathrm{fu}}=C_{E} \times f_{\mathrm{fu}}^{*} \text {, }
$$

with

$$
f_{\mathrm{fu}}^{*}=\overline{f_{\mathrm{fu}}}-3 \times \sigma,
$$

where $\overline{f_{\mathrm{fu}}}$ is the mean value of the ultimate tensile strength, $f_{\mathrm{fu}}^{*}$ is the characteristic value of this ultimate tensile strength, $\sigma$ is its standard deviation, and $C_{E}$ is a reduction coefficient depending on the fiber type and considering environmental effects on FRP ageing.

This approach is called "semiprobabilistic" in the sense that the safety margins are taken by considering a distance of three times standard deviation $\sigma$ from the mean performance (the "probabilistic part" of the approach) and 


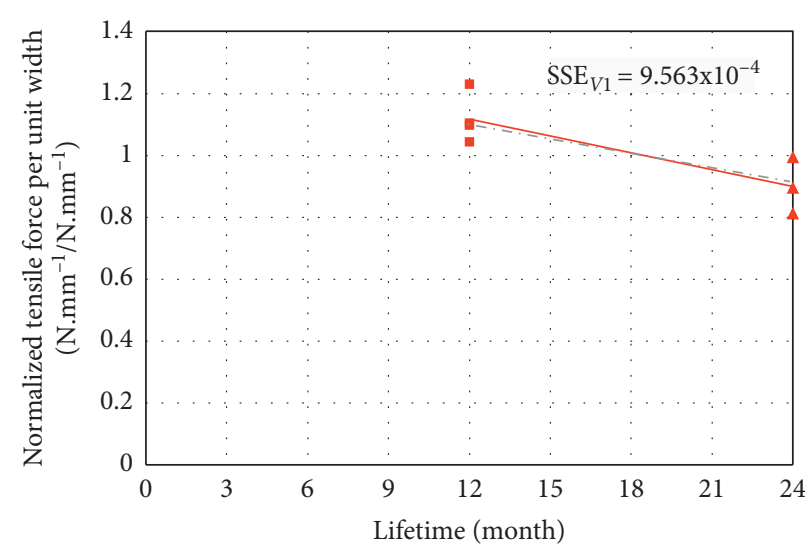

- $\Delta$ Mean degradation path for $V 1$

_. - ED model for $V 1$

(a)

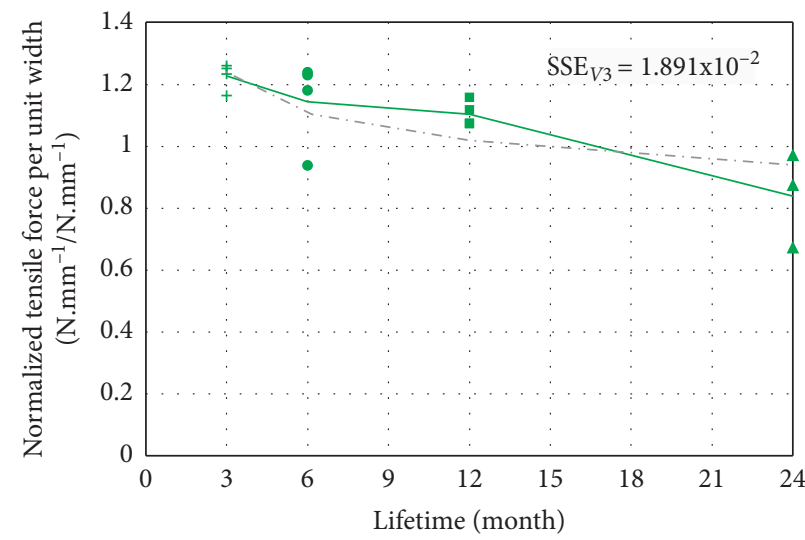

ta- Mean degradation path for $V 3$

... $\quad$ ED model for $V 3$

(c)

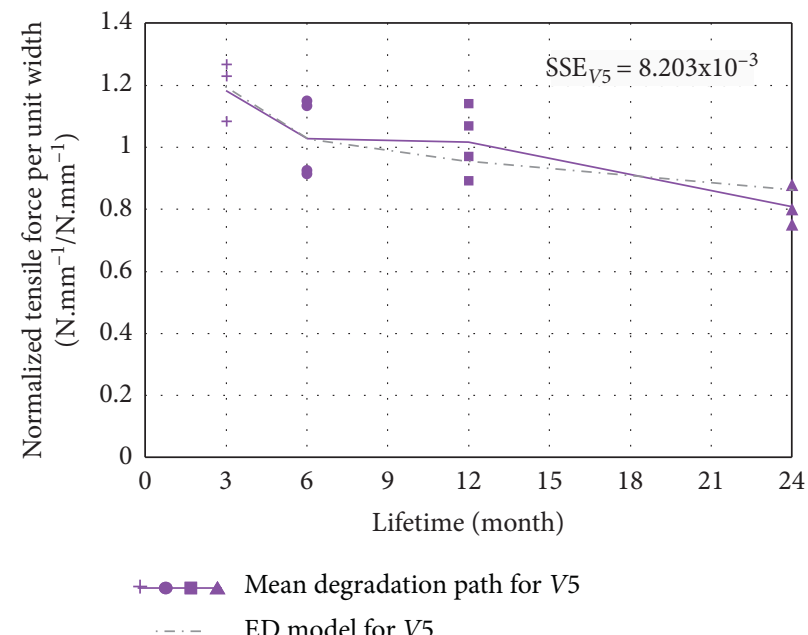

(e)

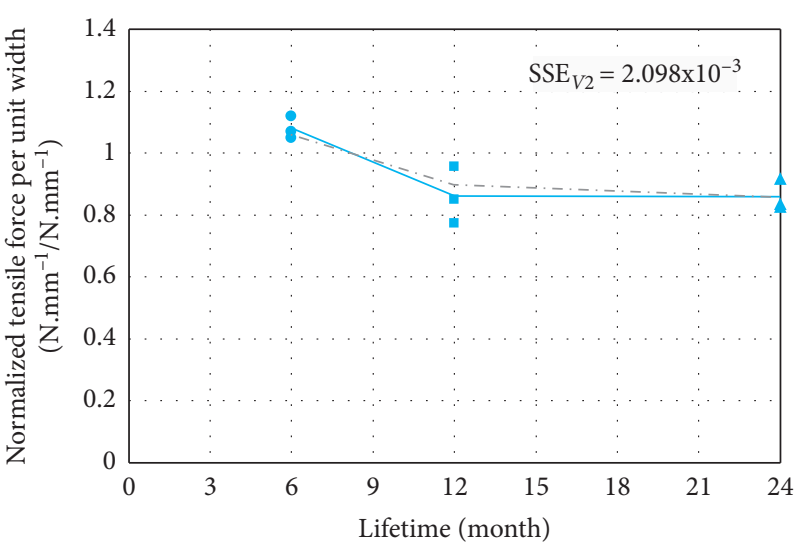

- $\triangle$ Mean degradation path for $V 2$

... ED model for $V 2$

(b)

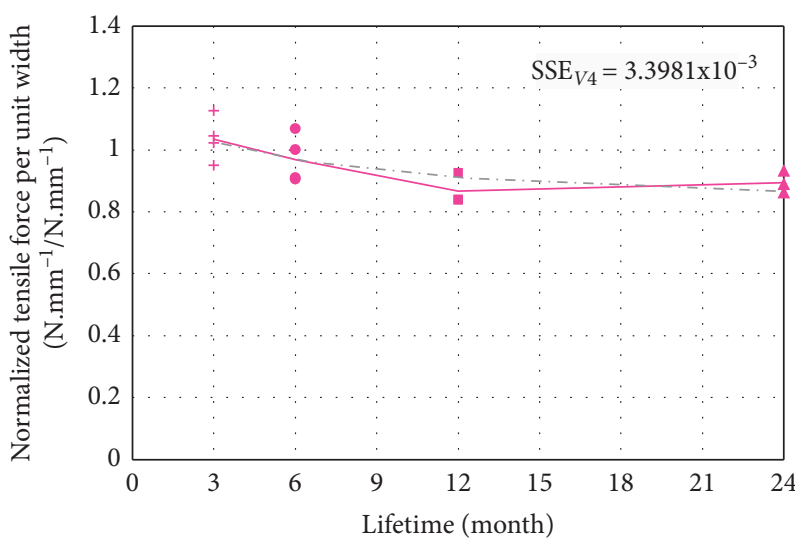

t.- Mean degradation path for $V 4$

ED model for $V 4$

(d)

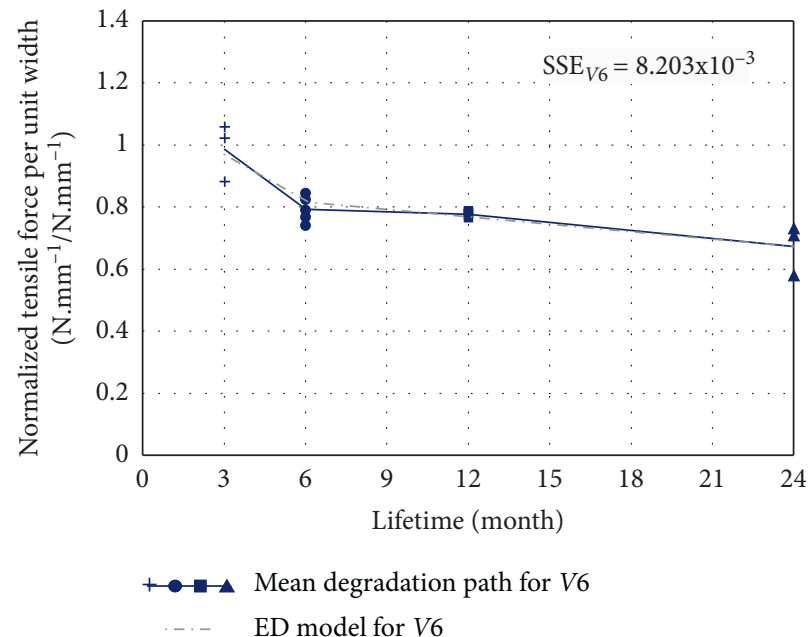

(f)

FIGURE 6: Comparison between experimental and $\mathrm{ED}$ model values under different ageing conditions: (a) $V 1\left(20^{\circ} \mathrm{C}-50 \% \mathrm{RH}\right)$ ageing condition, (b) $V 2\left(20^{\circ} \mathrm{C}-100 \% \mathrm{RH}\right)$ ageing condition, (c) $V 3\left(60^{\circ} \mathrm{C}-50 \% \mathrm{RH}\right)$ ageing condition, (d) $V 4\left(40^{\circ} \mathrm{C}-100 \% \mathrm{RH}\right)$ ageing condition, (e) V5 $\left(60^{\circ} \mathrm{C}-75 \% \mathrm{RH}\right)$ ageing condition, and (f) $V 6\left(60^{\circ} \mathrm{C}-100 \% \mathrm{RH}\right)$ ageing condition. 
that it can provide a deterministic value of the lifetime $t_{f}$. This latter is estimated, physically speaking, as the time required to degrade the tensile capacity value from its initial value $f_{\mathrm{fu}}^{*}(0)$ to the design value $f_{\mathrm{fu}}$. In other words, the time at which $f_{f}\left(t_{f}\right) / f_{\text {fu }}^{*}(0)=C_{E}$ (where $f_{f}(t)$ represents the value of FRP tensile capacity as a function of time).

As equation (21), from ACI 440 2R-17 [30] code, relies on the assumption that the statistical distribution of $f_{f}$ is normal, it can be stated that the calculated deterministic value of lifetime $t_{f}$ can be guaranteed with a level of confidence $\ell_{c}$. This level of confidence, not to be confused with "confidence interval," can be calculated rewriting equations (20) and (21) into

$$
\frac{f_{\mathrm{fu}}}{\overline{f_{\mathrm{fu}}}}=C_{E} \times(1-3 \times \mathrm{CoV})=1-\left(\frac{1-C_{E}}{\mathrm{CoV}}+3 \times C_{E}\right) \times \mathrm{CoV},
$$

where $\mathrm{CoV}=\left(\sigma / \overline{f_{\mathrm{fu}}}\right)$ is the coefficient of variation of the tensile capacity.

And, next, we define

$$
\ell_{c}=\Phi\left[-\left(\frac{1-C_{E}}{\mathrm{CoV}}+3 \times C_{E}\right)\right]
$$

where $\Phi(\cdot)$ is the CDF of the standard normal distribution.

For instance, if $\mathrm{CoV}=10 \%$ and $C_{E}=0.85, \ell_{c}=\Phi$ [-4.05] is equal to $2.56 .10^{-5}$. It is statistically equivalent to the probability/risk that the tensile capacity per unit width is lower than its design value $f_{\mathrm{fu}}$.

Our approach has more potential because it provides not only one value of lifetime with a prefixed level of confidence but also, instead, the full description of the statistical distribution of the lifetime through the use of equation (9). If one focusses on the degradation of the normalized performance, $f_{f}(t) / f_{f}(0)$, the threshold value $\omega$ will have to be equal here to $C_{E} \times(1-3 \times \mathrm{CoV}) \cdot f_{f}(0)$ is the initial value of tensile capacity, which may actually show a certain amount of statistical variability.

Even if the other design codes, TR55 [31], Fib Bulletin 40 [32], and AFGC guide [21], may rely on different safety concepts (assumptions and formalisms), our approach can be applied to these specific guidelines straightforwardly. The formalisms of the other design codes, with information about the levels of confidence and with the values of the threshold to choose for our fully probabilistic approach, are summed up in Table 3. It is needed to point out that the expression of threshold $\omega$ for AFGC guides does not integrate the coefficient of variation $(\mathrm{CoV})$ of performance. It is rather confusing, and the validity of such expression should be reconsidered, in our opinion.

4.4. Probabilistic Analysis of Lifetime. TED degradation process is being determined (see Section 4.1), and it is thus possible to draw the reliability functions of FFRP laminates as a function of time with specified values of the threshold $\omega$ through equation (9). It is also possible to deduce indicators of lifetime (equations (10) and (11)) of FFRP laminates under a specific environmental condition of temperature and relative humidity. As already mentioned in Section 4.3, it is reminded that the condition $V 1\left(T=20^{\circ} \mathrm{C}, \mathrm{RH}=50 \%\right)$ is considered as the service condition. However, since the expression of the degradation rate $\mu$ as a function of $T$ and $\mathrm{RH}$ is embedded in the TED process set of parameters, any environmental conditions could be used.

No values of safety reduction and environmental coefficients found in the design recommendations mentioned below are proposed specifically for FFRP laminates. Thus, in the following, the coefficients specified within the design codes for carbon (CFRP) and E-glass (GFRP) fiber reinforced polymers will be considered for reference in a first approach. Clearly, the probabilistic indicators of FFRP laminates lifetime are estimated using either CFRP or GFRP coefficients provided by ACI 440 2R-17 [30], TR55 [31], Fib Bulletin 40 [32], and AFGC [21] guides.

All design codes are less conservative in the case of CFRP compared to GFRP, due to the fact that the variability (i.e., coefficient of variation, $\mathrm{CoV}$ ) of the latter is higher than the first ones. That leads, as an illustration with the ACI 440 2R17 [30] approach, to a stronger reduction of characteristic tensile strength $f_{\mathrm{fu}}^{*}$ (see equations (20) and (21)) for GFRP laminates $\left(C_{E}\right.$ of E-glass $\left.=0.65\right)$ than for CFRP ones $\left(C_{E}\right.$ of carbon $=0.85)$. For the estimation of probabilistic indicators of FFRP laminates, the same value of $\mathrm{CoV}$ calculated from our experimental observations, $\mathrm{CoV}=7.6 \%$, is used for both E-glass and carbon fibers. Taking into account the remark above about the difference in reduction factors, the lifetime indicators (MTTF and 10\%-quantile) will be bounded by a lower value provided by the less conservative case using the coefficients of CFRP and an upper one by the most conservative using the coefficients of GFRP.

Expressions derived for maximum allowable performance (threshold) $\omega$ values are given for CFRP and GFRP laminate types in the fifth column of this table.

Estimated indicators of FFRP lifetime (MMTF and 10\%quantile), for the reference condition $V 1$, are shown in Table 4 and reported in Figures 7(a)-7(f).

Depending on the considered design codes, the failure threshold $\omega$ varies greatly (see the third column of Table 4), which directly leads to obvious differences in lifetime. The reliability functions of carbon and E-glass fiber composites relying, respectively, either on ACI 440 2R-17 [30], TR55 [31], Fib Bulletin 40 [32], or AFGC [21] guides are shown in Figures $7(\mathrm{a})-7(\mathrm{~d})$. We must bear in mind that these reliability functions are obtained integrating the safety coefficients; thus, one should not be confused by the fact that CFRP laminates seem less reliable than GFRP ones. It is just that the codes are more conservative in the case of the latter type of fibers, as explained above. We remark, to illustrate this point, that the ratios of MMTF values between GFRP and CFRP are equal to 2.0 for both ACI 440 2R-17 [30] and TR55 [31], to 3.7 for Fib Bulletin 40 [32] and to 1.3 for AFGC guide [21]. For Fib Bulletin 40 [32], the great difference between the MTTF of GFRP and CFRP laminates is due to the large discrepancy between the associated values of the partial environmental reduction factors $\eta_{\mathrm{env}, t}$ (4.16 for GFRP 


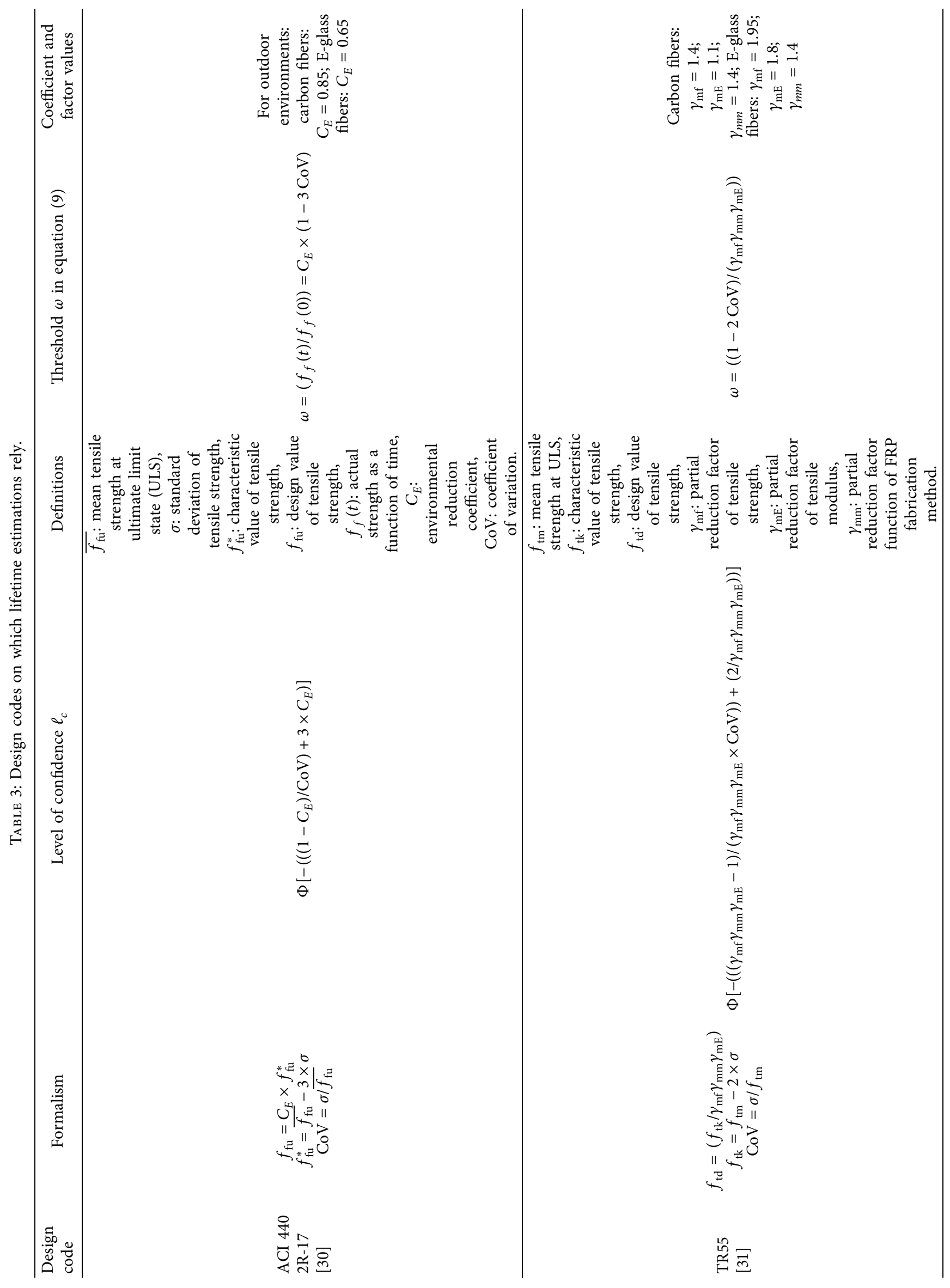




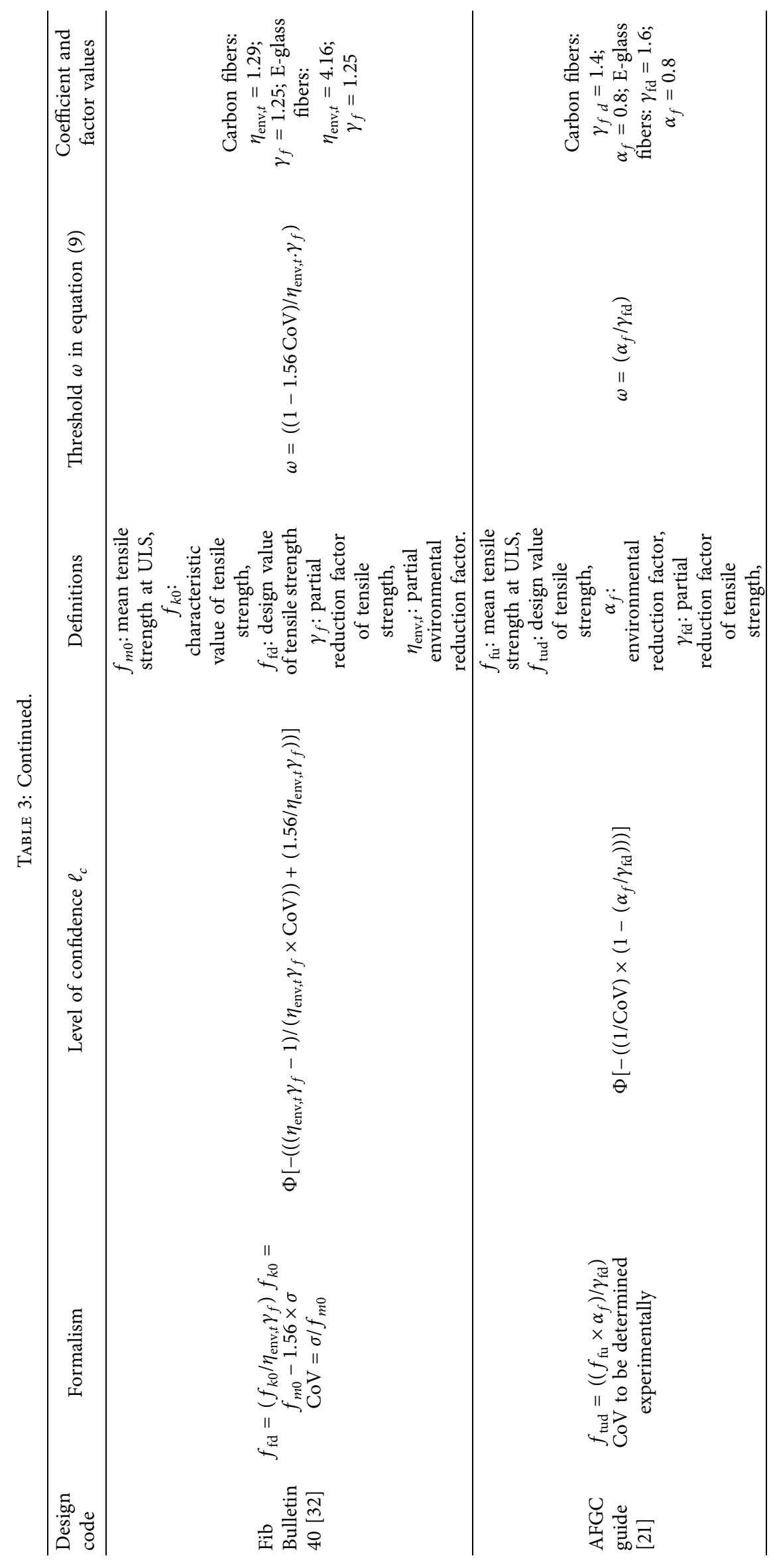


TABLE 4: Estimated lifetime (MTTF and 10\%-quantile), threshold, and level of confidence $\ell_{c}$ for FFRP laminates exposed to the reference condition $V 1\left(20^{\circ} \mathrm{C}-\mathrm{RH} 100 \%\right)$.

\begin{tabular}{|c|c|c|c|c|c|c|}
\hline \multirow[t]{2}{*}{ Design code } & & \multirow[t]{2}{*}{ Coefficient values } & \multirow[t]{2}{*}{ Threshold $\omega^{*}$} & \multicolumn{2}{|c|}{$\begin{array}{l}\text { Estimated lifetime } \\
\text { (month/year) }\end{array}$} & \multirow[t]{2}{*}{ Level of confidence $\ell_{c}$} \\
\hline & & & & MTTF & 10\%-quantile & \\
\hline \multirow[b]{2}{*}{ ACI 440 2R-17 [30] } & \multirow[b]{2}{*}{ With } & $C_{E}=0.85$ (corresponding to carbon fibers coef.) & 0.656 & $72.1 / 6$ & $44.9 / 3.7$ & $3.039 e-06$ \\
\hline & & $C_{E}=0.65$ (corresponding to E-glass fibers coef.) & 0.502 & $\begin{array}{c}146.4 / \\
12.2\end{array}$ & $87.0 / 7.3$ & $2.778 e-11$ \\
\hline \multirow{2}{*}{ TR55 [31] } & \multirow{2}{*}{ With } & $\gamma_{\mathrm{mf}}=1.4 ; \gamma_{\mathrm{mE}}=1.1 ; \gamma_{\mathrm{mm}}=1.4$ (carbon fibers coef. $)$ & 0.393 & $\begin{array}{c}213.4 / \\
17.8\end{array}$ & $133.3 / 11.1$ & $7.163 e-16$ \\
\hline & & $\gamma_{\mathrm{mf}}=1.95 ; \gamma_{\mathrm{mE}}=1.8 ; \gamma_{\mathrm{mm}}=1.4$ (E-glass fibers & 0.173 & $\begin{array}{c}435.0 / \\
36.3 \\
\end{array}$ & $282.6 / 23.6$ & $6.628 e-28$ \\
\hline \multirow{2}{*}{ Fib Bulletin 40 [32] } & \multirow{2}{*}{ With } & $\eta_{\mathrm{env}, t}=1.29 ; \gamma_{f}=1.25$ (carbon fibers coef.) & 0.547 & $\begin{array}{c}119.5 / \\
10.0\end{array}$ & $73.9 / 6.2$ & $1.220 e-09$ \\
\hline & & $\eta_{\mathrm{env}, t}=4.16 ; \gamma_{f}=1.25$ (E-glass fibers coef.) & 0.169 & $\begin{array}{c}440.4 / \\
36.7\end{array}$ & $281.2 / 23.4$ & $4.257 e-28$ \\
\hline \multirow[b]{2}{*}{ AFGC guide [21] } & \multirow[b]{2}{*}{ With } & $\gamma_{\mathrm{fd}}=1.4 ; \alpha_{f}=0.8$ (carbon fibers coef.) & 0.571 & $110.4 / 9.2$ & $66.7 / 5.6$ & $8.547 e-09$ \\
\hline & & $\gamma_{\mathrm{fd}}=1.6 ; \alpha_{f}=0.8$ (E-glass fibers coef.) & 0.500 & $\begin{array}{c}146.6 / \\
12.2\end{array}$ & $89.8 / 7.5$ & $2.369 e-11$ \\
\hline
\end{tabular}

*From expressions in the $5^{\text {th }}$ column of Table 3 .

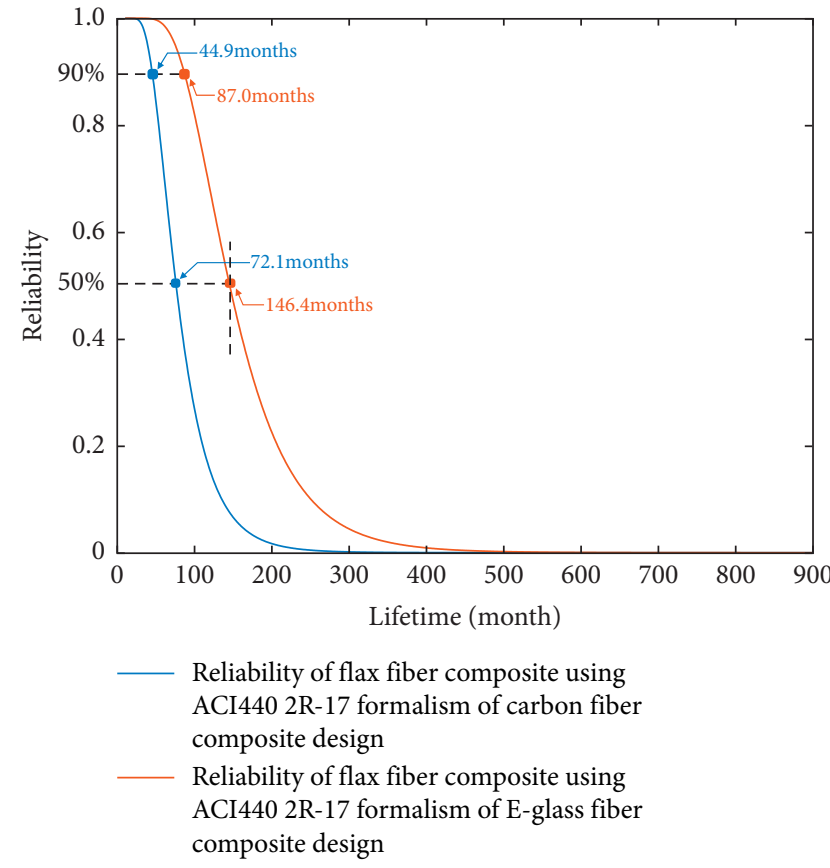

(a)

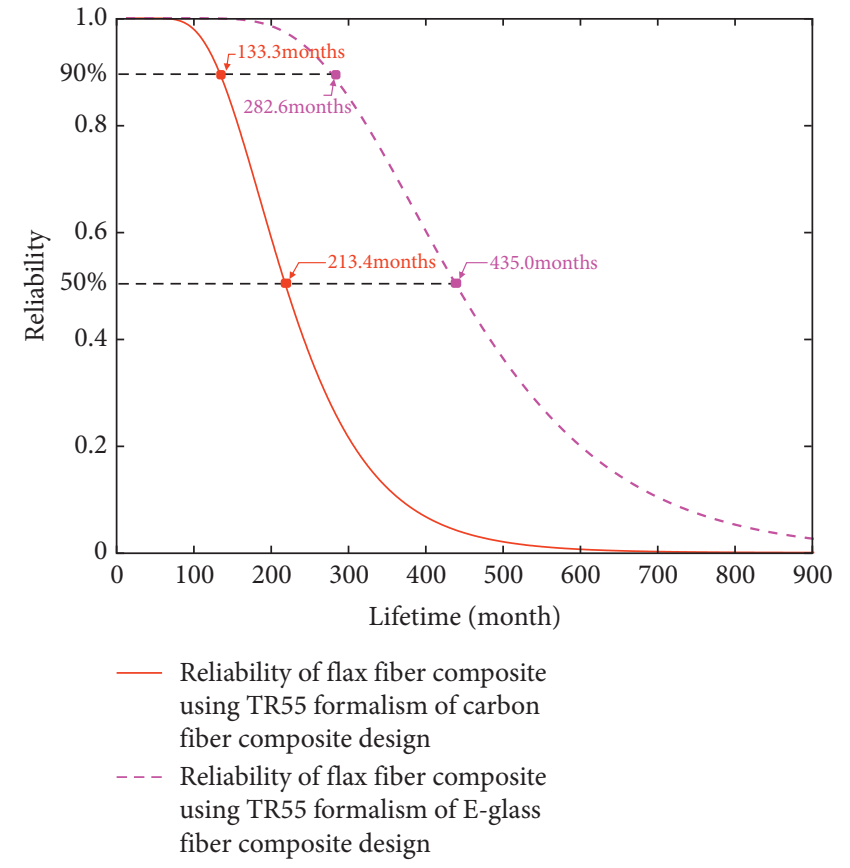

(b)

Figure 7: Continued. 


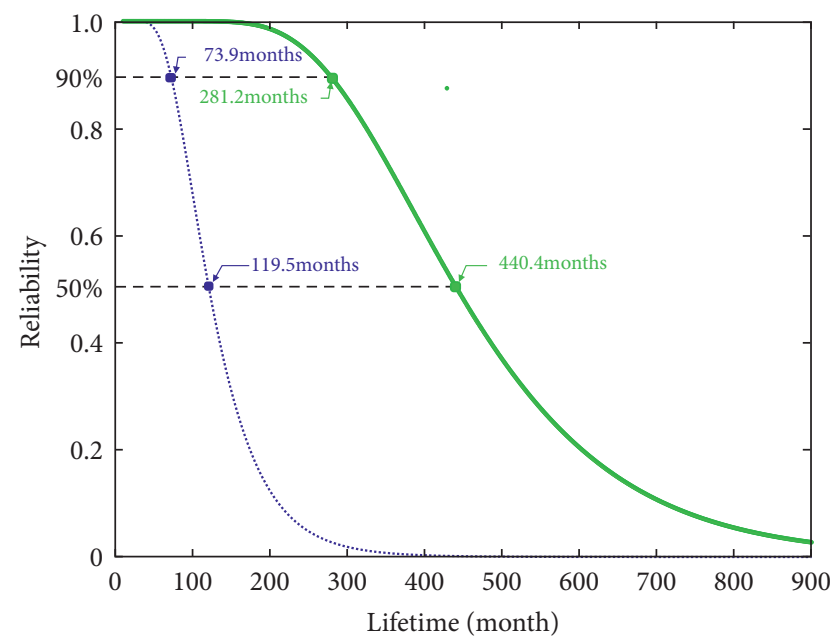

Reliability of flax fiber composite using Fib 40 formalism of carbon fiber composite design

- Reliability of flax fiber composite using Fib40 formalism of E-glass fiber composite design

(c)

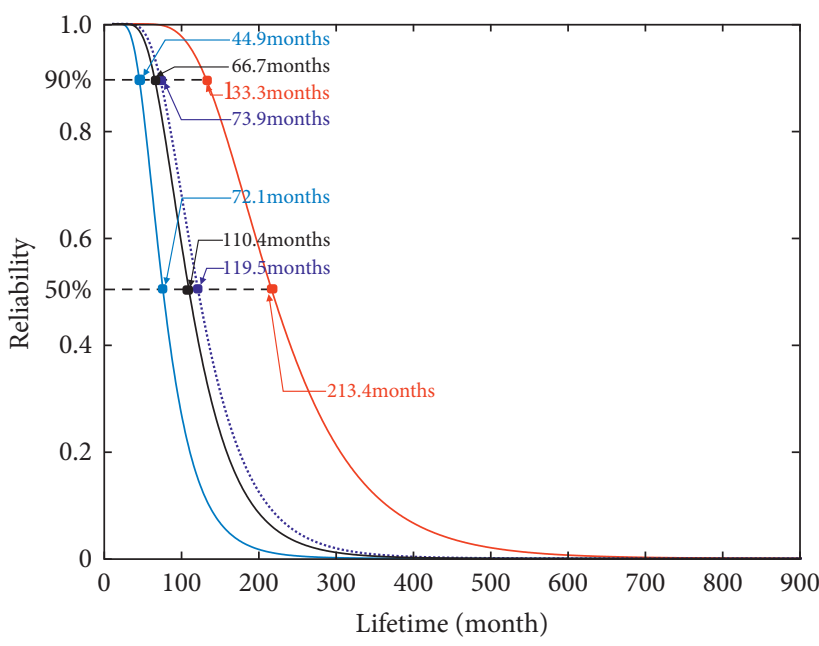

_ Reliability of flax fiber composite using ACI 440 2R-17 formalism of carbon fiber composite design

— Reliability of flax fiber composite using TR55 formalism of carbon fiber composite design
Reliability of flax fiber composite using Fib40 formalism of carbon fiber composite design

- Reliability of flax fiber composite using AFGC guide formalism of carbon fiber composite design

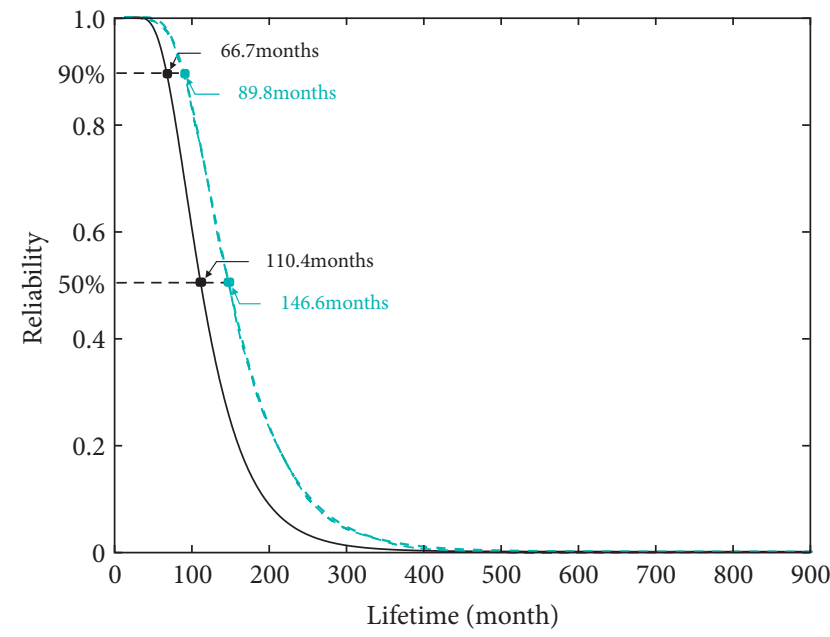

_ Reliability of flax fiber composite using AFGC guide formalism of carbon fiber composite design

- - - Reliability of flax fiber composite using AFGC guide formalism of E-glass fiber composite design

(d)

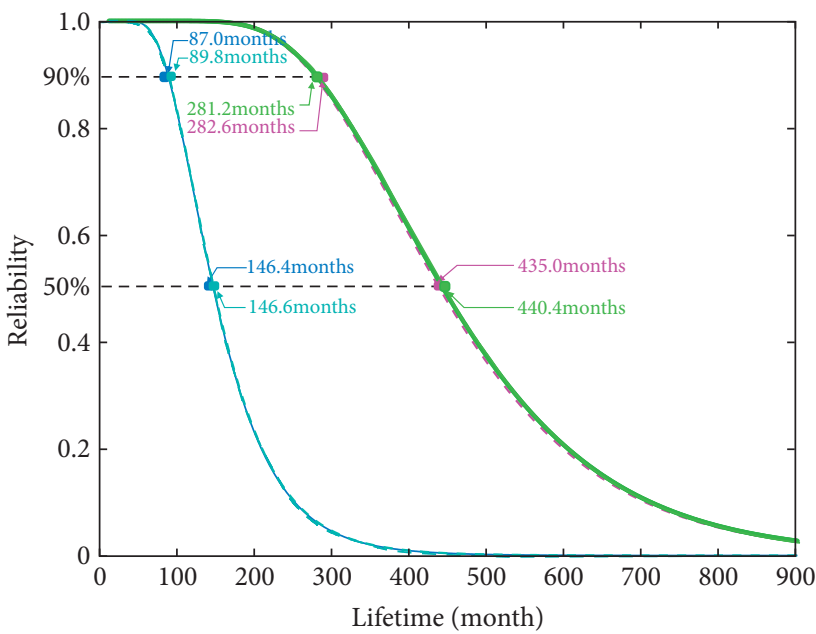

- Reliability of flax fiber composite using ACI 440 2R-17 formalism of E-glass fibers

_ Reliability of flax fiber composite using Fib40 formalism of E-glass fibers - Reliability of flax fiber
composite using TR55 formalism of E-glass fibers

- Reliability of flax fiber composite using AFGC guide formalism of E-glass fibers

(e)

(f)

FIGURE 7: Reliability functions of FFRP composites relying on different design codes: (a) reliability of carbon and E-glass fibers relying on ACI 440 2R-17 [30], (b) reliability of carbon and E-glass fibers relying on TR55 [31], (c) reliability of carbon and E-glass fibers relying on Fib Bulletin 40 [32], (d) reliability of carbon and E-glass fibers relying on AFGC guide [21], (e) reliability of carbon fibers relying on the different design codes (ACI 440 2R-17 [30], TR55 [31], Fib Bulletin 40 [32], and AFGC guide [21]), and (f) reliability of E-glass fibers relying on the different design codes (ACI 440 2R-17 [30], TR55 [31], Fib Bulletin 40 [32], and AFGC guide [21]). 


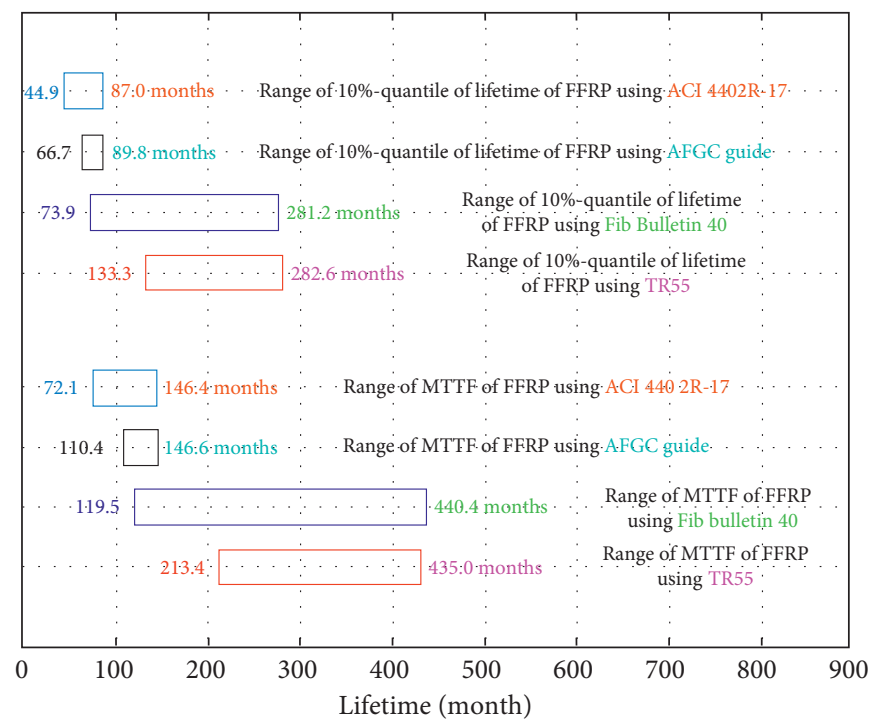

FIGURE 8: Estimations of lifetime indicator intervals for FFRP composites relying on different design codes (lower bounds are provided using the coefficients of CFRP and upper bounds are provided using those of GFRP).

TABLe 5: Parameter requirements to reach target lifetime.

\begin{tabular}{|c|c|c|c|c|c|c|}
\hline \multicolumn{7}{|c|}{ Design codes $\longrightarrow$} \\
\hline $\begin{array}{r}\downarrow \text { Targe } \\
\text { MTTF (years) }\end{array}$ & $\begin{array}{l}\text { ifetime } \\
\begin{array}{l}\text { 10\%-quantile } \\
\text { (years) }\end{array}\end{array}$ & $\begin{array}{l}\downarrow \text { Corresponding } \\
\text { threshold } \omega\end{array}$ & $\begin{array}{c}\text { ACI } 4402 \mathrm{R}-17 \\
{[30]}\end{array}$ & $\begin{array}{c}\text { TR55 [31] } \\
\left(\gamma_{\mathrm{mE}}=1.8 ; \gamma_{\mathrm{mm}}=1.4\right)\end{array}$ & $\begin{array}{l}\text { Fib Bulletin } 40 \\
{[32]\left(\gamma_{f}=1.5\right)}\end{array}$ & $\begin{array}{l}\text { AFGC guide [21] } \\
\qquad\left(\alpha_{f}=0.8\right)\end{array}$ \\
\hline 5.0 & 3.0 & 0.686 & $C_{E}=0.89$ & $\gamma_{\mathrm{mf}}=0.49$ & $\eta_{\mathrm{env}, t}=1.03$ & $\gamma_{\mathrm{fd}}=1.17$ \\
\hline 8.0 & 5.0 & 0.600 & $C_{E}=0.78$ & $\gamma_{\mathrm{mf}}=0.56$ & $\eta_{\mathrm{env}, t}=1.17$ & $\gamma_{\mathrm{fd}}=1.33$ \\
\hline 10.0 & 6.3 & 0.550 & $C_{E}=0.71$ & $\gamma_{\mathrm{mf}}=0.61$ & $\eta_{\mathrm{env}, t}=1.28$ & $\gamma_{\mathrm{fd}}=1.46$ \\
\hline 15.0 & 9.7 & 0.441 & $C_{E}=0.57$ & $\gamma_{\mathrm{mf}}=0.76$ & $\eta_{\mathrm{env}, t}=1.60$ & $\gamma_{\mathrm{fd}}=1.82$ \\
\hline 15.5 & 10.0 & 0.432 & $C_{E}=0.56$ & $\gamma_{\mathrm{mf}}=0.78$ & $\eta_{\mathrm{env}, t}=1.64$ & $\gamma_{\mathrm{fd}}=1.86$ \\
\hline 20.0 & 13.0 & 0.353 & $C_{E}=0.46$ & $\gamma_{\mathrm{mf}}=0.95$ & $\eta_{\mathrm{env}, t}=2.00$ & $\gamma_{\mathrm{fd}}=2.27$ \\
\hline 22.9 & 15.0 & 0.310 & $C_{E}=0.40$ & $\gamma_{\mathrm{mf}}=1.08$ & $\eta_{\mathrm{env}, t}=2.27$ & $\gamma_{\mathrm{fd}}=2.58$ \\
\hline 25.0 & 16.4 & 0.283 & $C_{E}=0.37$ & $\gamma_{\mathrm{mf}}=1.19$ & $\eta_{\mathrm{env}, t}=2.49$ & $\gamma_{\mathrm{fd}}=2.83$ \\
\hline 30.4 & 20.0 & 0.223 & $C_{E}=0.29$ & $\gamma_{\mathrm{mf}}=1.51$ & $\eta_{\mathrm{env}, t}=3.16$ & $\gamma_{\mathrm{fd}}=3.59$ \\
\hline
\end{tabular}

vs. 1.29 for CFRP). On the opposite, the difference of MTTF between GFRP and CFRP laminates provided by AFGC guide [21] is very low. This is due to the low discrepancy of the partial reduction factor of tensile strength $\gamma_{\mathrm{fd}}$ (1.6 for GFRP vs. 1.4 for CFRP). Once again, the formalism of AFGC guide [21] should draw our attention since it seems to be not enough conservative for GFRP laminates.

In Figures 7(e) and 7(f), the reliability functions and associated lifetime indicators provided by the four design codes are compiled, respectively, for CFRP and GFRP laminates in single graph. Relying on the principle stated previously that FFRP reliability functions can be in a first approach considered bounded by CFRP (low limit) and GFRP (upper limit) reliability functions, Figure 7 is completed by Figure 8 which gives an overview of the bounds of MTTF and 10\%-quantile indicators of lifetime for FFRP laminates using formalisms of the four different design codes. This overview confirms the less conservativeness of ACI 440 2R-17 [30] and AFGC guides [21] design codes compared to Fib Bulletin 40 [31] and TR55 [32] ones. It also confirms the great discrepancy of the estimated values of lifetime indicators between the different design codes. For example, MTTF ranges from 72.1 months (6 years), for ACI 440 2R-17 [30] with CFRP coefficients, to 440.4 months (36.7 years), for Fib Bulletin 40 [31] with GFRP coefficients.

It must be concluded from what it is written above that the lack of dedicated coefficients for flax fiber laminates within the leading design codes makes it too imprecise for the estimation of their lifetime indicators. To address this shortcoming, we propose new coefficients specific for FFRP laminates for the four design codes; coefficients which are variable according to the target lifetime (examples for target MTTF or target $10 \%$-quantile values are presented in Table 5). In that way, we go further than existing codes that do not explicitly take into consideration different choices of design target lifetime. Based on our results and the estimated TED degradation process, the reduction coefficients for different target values of lifetime in the specific case of FFRP laminates are provided in Table 5. For the ACI 440 2R-17 code [30], the only parameter to determine is the 
environmental reduction coefficient $C_{E}$. For TR55 [31] recommendations, since we do not have significant information about the variation of tensile modulus with time, the partial reduction factor of the modulus $\gamma_{\mathrm{mE}}$ is taken to its more conservative value (i.e., $\gamma_{\mathrm{mE}}=1.8$ ) and the partial reduction factor $\gamma_{\mathrm{mm}}$ related to FRP fabrication method, being invariant between CFRP and GFRP, is taken at 1.4. Thus, we just have to determine the partial reduction factor of tensile strength $\gamma_{\mathrm{mf}}$. For Fib Bulletin 40 code [32], the parameter to determine is the environmental reduction factor $\eta_{\text {env, } t}$; the partial reduction factor of tensile strength is taken at the same values as for CFRP and GFRP laminates, i.e., $\gamma_{f}=1.25$. Finally, for AFGC guide [21], the only parameter to adjust is the partial reduction factor of tensile strength $\gamma_{f d}$; the environmental reduction factor is also taken at the same values as for CFRP and GFRP laminates, i.e., $\alpha_{f}=0.8=0.8$.

The threshold $\omega$ values corresponding to the target lifetime indicators are given in the third column of Table 5. We remind that this threshold is evaluated as the ratio between the performance at time $t$ and this performance at the initial time $\left(\omega=f_{f}(t) / f_{f}(0)\right)$. In other words, the threshold $\omega$ can be viewed as the percentage of the remaining performance after a given time of service. This threshold clearly decreases over time, and hence, the reduction coefficients have to be adjusted according to the target lifetime values. Thus, to be more conservative, for high values of target lifetime indicators, the $C_{E}$ coefficient of ACI 440 2R-17 rules has to be decreased. For the other coefficients $\gamma_{\mathrm{mf}}, \eta_{\mathrm{env}, t}$, and $\gamma_{\mathrm{fd}}$, brought to the denominator, those have to be increased with increasing target lifetime values.

To complete, we need to keep in mind that the provided values of the reduction coefficients $C_{E}, \gamma_{\mathrm{mf}}, \eta_{\mathrm{env}, t}$, and $\gamma_{\mathrm{fd}}$ are independent of (or available whatever is) the $\mathrm{CoV}$ of the FFRP laminate tensile strength. Only the corresponding threshold $\omega$ will change according to the $\mathrm{CoV}$ value.

\section{Conclusions}

The main motivation of this study was to propose extensions to international guides dedicated to the design of FRP strengthened reinforced concrete structures for the special case of FRPs made of biosourced resin and flax fibers. This biocomposite FFRP represents a sustainable alternative to glass or carbon FRPs. Due to the impossibility to carry out tests under service conditions with the aim to guarantee safe performances for several years or decades, accelerated degradation tests have to be considered. FFRP laminates were subjected to six accelerated ageing conditions, and their mechanical performances were monitored over a period of 24 months. The degradation was stimulated by two stress variables, temperature and relative humidity, which is rarely seen in experimental/theoretical papers from the literature. In the present study, a lifetime prediction approach based on the Tweedie exponential dispersion (TED) model combined with the generalized Eyring model (GEM) was calibrated according to the experimental observations. The TED model was chosen because it is more versatile and particularly accurate for complicated degradation mechanisms, which was the case in our study. The GEM enables to consider the individual and coupling effects of temperature and relative humidity on the parameters of the TED process model. In addition, a correction was made to take into consideration the effect of the postcuring phenomenon of the epoxy matrix. To our knowledge, combination of the TED model with a two-stress variable acceleration function, such as GEM, has not yet been proposed by our community.

To go back to our main objective, new reduction coefficients for FFRP laminates have been proposed in order to update four of the international leading design codes (ACI 440 2R-17 [30], TR55 recommendations [31], Fib Bulletin 40 [32], and AFGC guide [21]). An originality of the present approach is to derive reduction coefficients adapted to a target lifetime from a probabilistic perspective, which is not proposed by current design codes. It is a fundamental approach in order to avoid noneconomic overdesign while still ensuring safety requirements.

Regarding the perspectives of this study, we are deeply convinced that international design codes should adopt approaches considering varying reduction coefficient defined for a specific lifetime. Considering the particular case of TR55 [31] guide, calibration of reduction coefficient $\gamma_{\mathrm{mE}}$ should be explored since this parameter, which has not been monitored in the present study, varies over time. This could provide a more optimal design for FRP strengthening systems. With a wider perspective, we are also confident that the global approach, which consists in considering the probabilistic analysis of long-term performances of FRPs through accelerated degradation tests and stochastic process modeling, deserves to be extended to other loading contexts such as fatigue lifetime estimation [33] or other mechanical properties such as toughness [34]. The mathematical formalisms and their algorithmic counterparts have been made available by the scientific community.

\section{Data Availability}

The data used to support the findings of this study are available from the corresponding author on request.

\section{Disclosure}

All the authors have participated in (a) conception and design, or analysis and interpretation of the data; (b) drafting the article or revising it critically for important intellectual content; and (c) approval of the final version.

\section{Conflicts of Interest}

The authors declare that they have no conflicts of interest.

\section{Acknowledgments}

The authors would like to acknowledge the great support provided by the ANR (Agence Nationale de la Recherche) through contract no. ANR-15-CE22-0007-04- "MICRO: Matériaux Innovants Composites pour la Réparation d'Ouvrage", by National Natural Science Foundation of 
China (71861011), and by Jiangxi Provincial Postdoctoral Research Funding Project (2019KY35).

\section{References}

[1] L. Yan, N. Chouw, and K. Jayaraman, "Flax fibre and its composites-a review," Composites Part B: Engineering, vol. 56, pp. 296-317, 2014.

[2] H. Jariwala and P. Jain, "A review on mechanical behavior of natural fiber reinforced polymer composites and its applications," Journal of Reinforced Plastics and Composites, vol. 38, no. 10, pp. 441-453, 2019.

[3] A. Le Duigou, P. Davies, and C. Baley, "Interfacial bonding of flax fibre/poly (l-lactide) bio-composites," Composites Science and Technology, vol. 70, no. 2, pp. 231-239, 2010.

[4] D. Scida, M. Assarar, C. Poilâne, and R. Ayad, "Influence of hygrothermal ageing on the damage mechanisms of flax-fibre reinforced epoxy composite," Composites Part B: Engineering, vol. 48, pp. 51-58, 2013.

[5] A. Singh, S. Das, and E.-M. Craciun, "Effect of thermomechanical loading on an edge crack of finite length in an infinite orthotropic strip," Mechanics of Composite Materials, vol. 55, no. 3, pp. 285-296, 2019.

[6] D. Bigaud and O. Ali, "Time-variant flexural reliability of RC beams with externally bonded CFRP under combined fatiguecorrosion actions," Reliability Engineering \& System Safety, vol. 131, pp. 257-270, 2014.

[7] M. A. G. Silva, B. S. da Fonseca, and H. Biscaia, "On estimates of durability of FRP based on accelerated tests," Composite Structures, vol. 116, no. 1, pp. 377-387, 2014.

[8] M. Quiertant, K. Benzarti, J. Schneider, F. Landrin, M. Landrin, and F. Boinski, "Effects of ageing on the bond properties of carbon fiber reinforced polymer/concrete adhesive joints: investigation using a modified double shear test," Journal of Testing and Evaluation, vol. 45, no. 6, pp. 1920-1932, 2017.

[9] K. Benzarti, S. Chataigner, M. Quiertant, C. Marty, and C. Aubagnac, "Accelerated ageing behaviour of the adhesive bond between concrete specimens and CFRP overlays," Construction and Building Materials, vol. 25, no. 2, pp. 523538, 2011.

[10] Z.-S. Ye and M. Xie, "Stochastic modelling and analysis of degradation for highly reliable products," Applied Stochastic Models in Business and Industry, vol. 31, no. 1, pp. 16-32, 2015.

[11] W.-A. Yan, B.-W. Song, G.-L. Duan, and Y.-M. Shi, "Realtime reliability evaluation of two-phase wiener degradation process," Communications in Statistics-Theory and Methods, vol. 46, no. 1, pp. 176-188, 2017.

[12] W. Yan, D. Bigaud, N. M. Chaibati, and L. Izoret, "Optimization of accelerated destructive degradation testing of cementitious materials for their performances qualification under aggressive environments: the case of carbonation," Mathematical Problems in Engineering, vol. 2020, Article ID 5295627, 19 pages, 2020.

[13] H. Gao, L. Cui, and Q. Dong, "Reliability modeling for a twophase degradation system with a change point based on a Wiener process," Reliability Engineering \& System Safety, vol. 193, Article ID 106601, 2020.

[14] L. Naipeng, G. Nagi, and L. Yaguo, "Remaining useful life prediction of machinery under time-varying operating conditions based on a two-factor state-space model," Reliability Engineering \& System Safety, vol. 186, pp. 88-100, 2019.

[15] Z. Chen, S. Li, and E. Pan, "Optimal constant-stress accelerated degradation test plans using nonlinear generalized wiener process," Mathematical Problems in Engineering, vol. 2016, Article ID 9283295, 11 pages, 2016.

[16] X. Liu, Z. Wu, D. Cui, B. Guo, and L. Zhang, "A modeling method of stochastic parameters' Inverse gauss process considering measurement error under accelerated degradation test," Mathematical Problems in Engineering, vol. 2019, Article ID 9752920, 11 pages, 2019.

[17] P. Qian, L. Hong, W. Chen, Y. Qian, Z. Wang, and H. Yao, "Optimization of the accelerated degradation test plan for electrical connector contact pairs based on a nonlinear wiener process," Mathematical Problems in Engineering, vol. 2020, Article ID 5828374, 16 pages, 2020.

[18] S.-T. Tseng and I.-C. Lee, "Optimum allocation rule for accelerated degradation tests with a class of exponentialdispersion degradation models," Technometrics, vol. 58, no. 2 , pp. 244-254, 2016.

[19] F. Duan and G. Wang, "Exponential-dispersion degradation process models with random effects and covariates," IEEE Transactions on Reliability, vol. 67, no. 3, pp. 1128-1142, 2018.

[20] S. Zhou and A. Xu, "Exponential dispersion process for degradation analysis," IEEE Transactions on Reliability, vol. 68, no. 2, pp. 398-409, 2019.

[21] AFGC: Association Française de Génie Civil, "Réparation et renforcement des structures en béton au moyen des matériaux composites," in Bulletin Scientifique et Technique de l'AFGC, p. 114, Springer, Berlin, Germany, 2011.

[22] ASTM D7565/D7565M-10(2017), "Standard test method for determining tensile properties of fiber reinforced," in Polymer Matrix Composites Used for Strengthening of Civil StructuresASTM International, West Conshohocken, PA, USA, 2017.

[23] Z. Chen, T. Xia, Y. Li, and E. Pan, "Tweedie exponential dispersion processes for degradation modeling, prognostic, and accelerated degradation test planning," IEEE Transactions on Reliability, vol. 69, no. 3, pp. 887-902, 2020.

[24] Z. Chen, E. Pan, T. Xia, and Y. Li, "Optimal degradationbased burn-in policy using Tweedie exponential-dispersion process model with measurement errors," Reliability Engineering \& System Safety, vol. 195, Article ID 10674, 2020.

[25] B. Jørgensen, The Theory of Exponential Dispersion Models, Chapman \& Hall, London, UK, (ISBN 10: 0412997118, ISBN 13: 9780412997112), 1998.

[26] L. Q. Hong and Z. S. Ye, "When is acceleration unnecessary in a degradation test?," Statistica Sinica, vol. 27, pp. 1461-1483, 2017.

[27] L. A. Escobar and W. Q. Meeker, "A review of accelerated test models," Statistical Science, vol. 21, no. 4, pp. 552-577, 2007.

[28] K. Benzarti, R. Chlela, W. Zombré, M. Quiertant, and L. Curtil, "Durability of flax/bio-based epoxy composites intended for structural strengthening," MATEC Web of Conferences, vol. 199, p. 07014, 2018.

[29] R. Chlela, W. Zombré, M. Quiertant, L. Curtil, and K. Benzarti, "Hygrothermal ageing of flax fibre reinforced composites intended for the strengthening of concrete structures," in Proceedings of the 9th International Conference on Fibre-Reinforced Polymer (FRP) Composites in Civil Engineering, pp. 242-249, Paris, France, July 2018.

[30] ACI Committee 440, Guide for the Design and Construction of Externally Bonded FRP Systems for Strengthening Concrete Structures, (ISBN: 9781945487590), p. 112, American Concrete Institute, Indianapolis, IN, USAAmerican Concrete Institute, 2017.

[31] C. Arya, J. Clarke, E. Kay, and P. O'regan, TR55 Design Guidance for Strengthening Concrete Structures Using Fibre 
Composite Materials: A Reviewp. 187, 3rd edition, Concrete Society, London, UK, 2012.

[32] Fib Bulletin 40, "FRP reinforcement in RC structures," Technical Report, p. 151, Fib Fédération Internationale du Béton, Lausanne, Switzerland, 2007.

[33] E.-K. Kim, H. Oh, and J. Sim, "Semiempirical methodology for estimating the service life of concrete deck panels strengthened with fiber-reinforced polymer," Mathematical Problems in Engineering, vol. 2014, Article ID 273693, 13 pages, 2014.

[34] S. Samborski, "Prediction of delamination front's advancement direction in the CFRP laminates with mechanical couplings subjected to different fracture toughness tests," Composite Structures, vol. 202, pp. 643-650, 2018. 